ESAIM: COCV 19 (2013) 317-336

DOI: $10.1051 / \mathrm{cocv} / 2012010$
ESAIM: Control, Optimisation and Calculus of Variations

www.esaim-cocv.org

\title{
ON TIME OPTIMAL CONTROL OF THE WAVE EQUATION, ITS REGULARIZATION AND OPTIMALITY SYSTEM*
}

\author{
Karl Kunisch ${ }^{1}$ AND Daniel Wachsmuth ${ }^{2}$
}

\begin{abstract}
An approximation procedure for time optimal control problems for the linear wave equation is analyzed. Its asymptotic behavior is investigated and an optimality system including the maximum principle and the transversality conditions for the regularized and unregularized problems are derived.
\end{abstract}

Mathematics Subject Classification. 49K20, 93C20, 35L05.

Received March 20, 2012. Revised February 23, 2012.

Published online June 21, 2012.

\section{INTRODUCTION}

This paper is devoted to the time optimal control problem

$$
\left\{\begin{array}{l}
\min \int_{0}^{\tau} \mathrm{d} t \\
\text { subject to } \tau \geq 0, \\
y_{t t}-\Delta y=\chi_{\omega} u \quad \text { in } \quad(0, \tau) \times \Omega, \\
y(0)=y_{1}, y_{t}(0)=y_{2}, y(\tau)=z_{1}, \quad y_{t}(\tau)=z_{2} \text { in } \Omega \\
y=0 \text { on } \Gamma, \\
\|u(t)\|_{L^{2}(\omega)} \leq \gamma, \quad \text { for a.e. } t \in(0, \tau)
\end{array}\right.
$$

Here, $\gamma>0$ is a fixed positive constant and $\Omega \subset \mathbb{R}^{n}$ with $n \in\{1,2,3\}$ is a fixed bounded domain with a $C^{2}$-boundary $\Gamma$. Further $\omega \subset \Omega$ is a measurable subset and $\chi_{\omega} u$ denotes the extension-by-zero operator from $\omega$ to $\Omega$. The initial and terminal states are fixed and - unless specified otherwise - are assumed to satisfy

$$
y_{1} \in H_{0}^{1}(\Omega), z_{1} \in H_{0}^{1}(\Omega), y_{2} \in L^{2}(\Omega), z_{2} \in L^{2}(\Omega),
$$

where, without loss of generality, $\left(y_{1}, y_{2}\right) \neq\left(z_{1}, z_{2}\right)$. We shall analyze a regularization scheme for $(\tilde{P})$. In the present work the procedure is used to derive an optimality system for $(\tilde{P})$. We have also used it as the basis for

Keywords and phrases. Time optimal control, wave equation, optimality condition, transversality condition.

* Partially supported by "Fonds zur Förderung der Wissenschaftlichen Forschung" under SFB 32, Mathematical Optimization and Applications in the Biomedical Sciences.

1 Institute for Mathematics and Scientific Computing, Heinrichstraße 36, 8010 Graz, Austria. karl.kunisch@uni-graz.at; http://www.kfunigraz.ac.at/imawww/kunisch

2 Johann Radon Institute for Computational and Applied Mathematics (RICAM), Austrian Academy of Sciences, Altenbergerstraße 69, 4040 Linz, Austria. daniel.wachsmuth@oeaw.ac.at;

http://www.ricam.oeaw.ac.at/people/page/wachsmuth 
methods to solve $(\tilde{P})$ numerically. This will be described in a follow-up paper. The optimality system that we derive is complete in the sense that it contains as many equations as unknowns. It consists of the primal and adjoint equations, the maximum principle and the transversality condition.

In [3] the geometric form of the transversality conditions in infinite dimensions is derived along the wellknown lines of Lee and Markus [15] in finite dimensions. It states that the terminal state of the adjoint equation is normal to a supporting hyperplane to the reachable cone at the optimal time and the state $\left(z_{1}, z_{2}\right)$. This form, however, does not appear to be applicable computationally. Rather we aim for an analytical form of the transversality condition as utilized e.g. in [10], page 88, in the case of time optimal control for ordinary differential equations. In the case of time optimal control for parabolic problems such a form was obtained by Barbu in [2]. The technique used there does not appear to be applicable for $(\tilde{P})$. It could be applied in the case where the terminal constraint $y_{t}(\tau)=z_{2}$ is not enforced and $\omega=\Omega$. Time optimal control for the wave equation was also investigated in the work of Fattorini [5,6], Gugat [8], Gugat and Leugering [9], and Krabs [12, 13]. However, the transversality condition is not addressed in these references.

Time-optimal control problems can be addressed alternatively by solving appropriately defined dual normoptimal control problems, which are parameterized by the time $\tau$, see e.g. [7,12]. If for some parameter value $\hat{\tau}$ the norm-optimal control satisfies $\|\hat{u}\|_{L^{\infty}\left(0, \hat{\tau} ; L^{2}(\omega)\right)}=\gamma$, then $(\hat{\tau}, \hat{u})$ is a solution of $(\tilde{P})$. However, this equivalence is established only for the special case $\omega=\Omega$. This is due to the fact that the analysis requires the controllability of the system in arbitrarily small time and the bang-bang property for all time-optimal controls. To the best of our understanding these requirements are well-established only for the case $\omega=\Omega$. Moreover, an example in [9] shows that the equivalence of time-optimal and norm-optimal control problems cannot be expected in the general case.

The focus of our work is set on obtaining the optimality system by means of a regularization procedure. This is intimately related to notions of controllability without constraints on the controls, controllability under constraints, and the bang-bang nature of optimal controls. While controllability without constraints on the controls is well understood for the wave equation, and some results which are relevant to the present work are summarized in Section 2.2, the other two topics are only well-studied in the case that $\omega=\Omega$. Any advance on these issues will also contribute to the understanding of the time optimal control problem.

The remainder of the paper is organized as follows. In Section 2.1 we introduce the abstract form of the wave equation and recall selected regularity results. Section 2.2 contains a discussion of controllability results as far as they are relevant for the present paper, we give a sufficient condition for the existence of feasible controls and for existence of a solution to $(\tilde{P})$.

In Section 3 we introduce a family of approximating problems and derive their optimality systems, including the maximum principle and the transversality condition. We also verify constancy of the Hamiltonian along optimal trajectories. Then convergence of the primal variables of the approximating problems to a solution of $(\tilde{P})$ is shown. Convergence of the adjoint variables is addressed in Section 4. To obtain the maximum principle and the transversality condition for $(\tilde{P})$ as the limit of the approximating family of equations additional assumptions are needed. We consider two different situations: either the case that the optimal control is bang-bang, or the case when some a-priori estimate on the family of approximating adjoint solutions holds. This latter condition is investigated numerically for a special case in Section 6. In a short Section 5 an adapted penalty technique is considered.

\section{Preliminaries}

\subsection{Abstract formulation}

Let us first set forth some concepts for the wave equation

$$
\left\{\begin{array}{l}
y_{t t}-\Delta y=\chi_{\omega} u \quad \text { in } \quad(0, \tau) \times \Omega, \\
y(0)=y_{1}, \quad y_{t}(0)=y_{2}, \\
y=0 \text { on } \Gamma,
\end{array}\right.
$$


with $\tau>0$ fixed, that will be relevant to our work. For certain purposes of treating time optimal problems it is convenient to perform a transformation of equation (2.1) to the fixed time interval

$$
I:=(0,1)
$$

For instance, this allows to consider numerical realizations with respect to a fixed reference domain. In addition, to express (2.1) in abstract form we introduce the operators

$$
\mathbf{A}:=\left(\begin{array}{cc}
0 & I \\
\Delta & 0
\end{array}\right), \quad \mathbf{B}:=\left(\begin{array}{c}
0 \\
\chi_{\omega}
\end{array}\right)
$$

and vectors

$$
\mathbf{y}_{0}:=\left(\begin{array}{l}
y_{1} \\
y_{2}
\end{array}\right), \mathbf{z}:=\left(\begin{array}{c}
z_{1} \\
z_{2}
\end{array}\right), \mathbf{y}(t):=\left(\begin{array}{c}
\mathbf{y}_{1}(t) \\
\mathbf{y}_{2}(t)
\end{array}\right) .
$$

Then the wave equation (2.1) can be expressed as the first-order evolution equation

$$
\begin{aligned}
\mathbf{y}_{t} & =\tau(\mathbf{A y}+\mathbf{B} u) \quad \text { on } I, \\
\mathbf{y}(0) & =\mathbf{y}_{0} .
\end{aligned}
$$

The components of the solution $\mathbf{y}$ of this equation fulfill $\left(\mathbf{y}_{1}\right)_{t}=\tau \mathbf{y}_{2}$ and $\left(\mathbf{y}_{2}\right)_{t}=\tau\left(\Delta \mathbf{y}_{1}+\chi_{\omega} u\right)$. For convenience of notation we introduce the function spaces

$$
Y^{s}= \begin{cases}H^{s}(\Omega) & 0 \leq s<1 / 2, \\ H^{s}(\Omega) \cap\left\{y:\left.y\right|_{\Gamma}=0\right\} & s \geq 1 / 2, \\ \left(Y^{-s}\right)^{*} & s<0\end{cases}
$$

and the associated vector-valued spaces, which take account of the regularity of the components of solutions $\mathbf{y}$ of $(2.2)$ :

$$
\mathbf{Y}^{s}:=Y^{s} \times Y^{s-1}
$$

The index $s$ indicates that the first component of the vector function $\mathbf{y} \in \mathbf{Y}^{s}$ is in $H^{s}(\Omega)$, whereas the second component is in $H^{s-1}(\Omega)$. Utilizing this notation the operator $\mathbf{A}$ is a continuous linear operator in the following sense

$$
\mathbf{A} \in \mathcal{L}\left(\mathbf{Y}^{s}, \mathbf{Y}^{s-1}\right)
$$

Moreover, the operator $\mathbf{B}$ has the property

$$
\mathbf{B} \in \mathcal{L}\left(L^{2}(\omega), \mathbf{Y}^{1}\right) .
$$

For existence and uniqueness of weak solutions of the system (2.2), we have the following well-known result, see e.g. [14].

Theorem 2.1. Let $\mathbf{y}_{0} \in \mathbf{Y}^{0}, u \in L^{2}\left(I ; L^{2}(\omega)\right)$ be given. Then the first-order equation (2.2) admits a unique very weak solution $\mathbf{y}$ satisfying

$$
\mathbf{y} \in C\left(\bar{I} ; \mathbf{Y}^{0}\right) .
$$

If in addition $\mathbf{y}_{0} \in \mathbf{Y}^{1}$ holds, then the first-order equation (2.2) admits a unique weak solution $\mathbf{y}$ that satisfies

$$
\mathbf{y} \in C\left(\bar{I} ; \mathbf{Y}^{1}\right), \mathbf{y}_{t} \in C\left(\bar{I} ; \mathbf{Y}^{0}\right) .
$$

If moreover $\mathbf{y}_{0} \in \mathbf{Y}^{2}, u_{t} \in L^{2}\left(I ; L^{2}(\omega)\right)$, then

$$
\mathbf{y} \in C\left(\bar{I} ; \mathbf{Y}^{2}\right), \mathbf{y}_{t} \in C\left(\bar{I} ; \mathbf{Y}^{1}\right) .
$$


It remains to transform the original time-optimal control problem $(\tilde{P})$ to the interval $I$. We define the set of admissible controls by

$$
U_{a d}:=\left\{u \in L^{\infty}\left(I ; L^{2}(\omega)\right): u(t) \in U \text { a.e. on } I\right\},
$$

with $U$ given by

$$
U=\left\{u \in L^{2}(\omega):\|u\|_{L^{2}(\omega)} \leq \gamma\right\} .
$$

Using the abstract operators introduced above, problem $(\tilde{P})$ can be expressed as

$$
\left\{\begin{array}{l}
\min \tau \\
\text { subject to } \tau \geq 0 \text { and } \\
\mathbf{y}_{t}=\tau(\mathbf{A y}+\mathbf{B} u) \text { on } I \\
\mathbf{y}(0)=\mathbf{y}_{0}, \quad \mathbf{y}(1)=\mathbf{z} \\
u \in U_{a d}
\end{array}\right.
$$

For the derivation of first-order necessary conditions as well as a discussion of controllability issues, we will frequently need the adjoint equation. It is defined as the evolution equation

$$
-\mathbf{p}_{t}=\tau \mathbf{A}^{*} \mathbf{p} \quad \text { on } I \text {. }
$$

Here $\mathbf{A}^{*}$ is given as adjoint of $\mathbf{A}$ :

$$
\mathbf{A}^{*}:=\left(\begin{array}{cc}
0 & \Delta \\
I & 0
\end{array}\right)
$$

Hence, equation (2.3) is a wave equation in the second coordinate $\mathbf{p}_{2}$ with $\partial_{t} \mathbf{p}_{2}=-\tau \mathbf{p}_{1}$ and

$$
\left(\mathbf{p}_{2}\right)_{t t}=\tau^{2} \Delta \mathbf{p}_{2} .
$$

It will be convenient to introduce the notation

$$
\mathbf{P}^{s}:=Y^{s-1} \times Y^{s}
$$

which will be used for $s=0,1,2$. The index $s$ with $\mathbf{Y}^{s}$ and $\mathbf{P}^{s}$ denotes the regularity of the wave function for the primal state $\mathbf{y}$ and the adjoint state $\mathbf{p}$, respectively. We may note that $\left(\mathbf{Y}^{s}\right)^{*}=\mathbf{P}^{(1-s)}$.

If the adjoint equation is complemented with a terminal condition $\mathbf{p}(1)=\hat{\mathbf{p}}$ with $\hat{\mathbf{p}} \in \mathbf{P}^{0}$, the adjoint equation is uniquely solvable with solution $\mathbf{p} \in C\left(\bar{I} ; \mathbf{P}^{0}\right)$. Moreover, one has regularity results analogous to those for the primal wave equation expressed in Theorem 2.1.

\subsection{Controllability and existence of time-optimal controls}

While the focus of this work lies on establishing an optimality system for the time optimal control problem with a regularization based approach, we also address the issue of existence to $(\mathrm{P})$, relying mostly on existing results which are adapted to the problem under consideration.

The question of existence of time-optimal controls is intimately linked to the question of (null)-controllability of the wave equation. The wave equation in its abstract form (2.2) is said to be null-controllable in time $\tau$ with controls in $L^{2}\left(I ; L^{2}(\omega)\right)$ if for every initial value $\mathbf{y}_{0} \in \mathbf{Y}^{1}$ there exists a control $u \in L^{2}\left(I ; L^{2}(\omega)\right)$ such that the solution $\mathbf{y}$ of $(2.2)$ satisfies $\mathbf{y}(1)=0$. It is well-known that null-controllability is equivalent to the following observability statement $[16,19]$ : there exists $c(\tau)>0$ such that every solution $\mathbf{p}$ of the adjoint equation

$$
-\mathbf{p}_{t}=\tau \mathbf{A}^{*} \mathbf{p} \quad \text { on } I
$$


satisfies

$$
\|\mathbf{p}(1)\|_{\mathbf{P}^{0}}^{2} \leq c(\tau) \tau\left\|\mathbf{B}^{*} \mathbf{p}\right\|_{L^{2}\left(I ; L^{2}(\omega)\right)}^{2} .
$$

Null-controllability of the wave equation with distributed (or internal) controls holds if the geometric control condition is satisfied: every ray of geometric optics propagating in the domain $\Omega$ hits the subset $\omega$ within time less than $\tau$, see [4]. Due to finite speed of propagation, null-controllability in general holds only if $\tau>\bar{\tau}$, where $\bar{\tau}$ solely depends on $\Omega, \omega$. For the special case $\omega=\Omega$, the wave equation is null-controllable for all $\tau>0$.

Remark 2.2. The additional factor $\tau$ in (2.5) appears due to the transformation $(0, \tau) \mapsto I$. The observability inequality in untransformed form reads $\|\phi(\tau)\|_{L^{2}(\Omega)}^{2}+\left\|\phi_{t}(\tau)\right\|_{H^{-1}(\Omega)}^{2} \leq c(\tau)\left\|\left.\phi\right|_{\omega}\right\|_{L^{2}\left(0, \tau ; L^{2}(\omega)\right)}^{2}$ for all solutions $\phi$ of the adjoint wave equation $\phi_{t t}-\Delta \phi=0$ in $(0, \tau) \times \Omega$.

The additional control constraints involved in the formulation of the time optimal control problem impose a serious difficulty. Naturally the question arises, whether for a given initial value $\mathbf{y}_{0} \in \mathbf{Y}^{1}$ there exists a control $u \in U_{a d}$ such that the solution $\mathbf{y}$ of $(2.2)$ satisfies $\mathbf{y}(1)=0$. Here, we have the following result [1,17]: if for all solutions $\mathbf{p}$ of the adjoint equation (2.4) the inequality

$$
\left\langle\mathbf{p}(0), \mathbf{y}_{0}\right\rangle_{\mathbf{P}^{0}, \mathbf{Y}^{1}}+\gamma \tau\left\|\mathbf{B}^{*} \mathbf{p}\right\|_{L^{1}\left(I ; L^{2}(\omega)\right)} \geq 0
$$

holds, then there exists a control $u \in U_{a d}$ steering the state $\mathbf{y}$ from $\mathbf{y}_{0}$ to $\mathbf{y}(1)=0$.

In the following, we will prove existence of feasible points for problem (P). We will show that for $\tau$ large enough, there is an admissible control $u$ such that all the constraints of $(\mathrm{P})$ are satisfied. The essential requirement is that the system is unrestricted null-controllable for some fixed time $\tau_{0}>0$. We will use a technique from [18], which allows us to provide an upper bound on the time $\tau$. This slightly extends [18], Theorem 4.2, where existence of time-optimal controls for $(\mathrm{P})$ is proven.

Proposition 2.3. Let $\mathbf{y}_{0}, \mathbf{z} \in \mathbf{Y}^{1}$ be given and let $\tau_{0}>0$ such that the wave equation is null-controllable, i.e. (2.5) holds with $c\left(\tau_{0}\right)$. Let $N>0$ be a natural number such that

$$
N \geq 2 c\left(\tau_{0}\right) \gamma^{-1} \max \left(\left\|\mathbf{y}_{0}\right\|_{\mathbf{Y}^{1}},\|\mathbf{z}\|_{\mathbf{Y}^{1}}\right) .
$$

Then there exists a feasible control $u$ for the time-optimal control problem, that drives the system from $\mathbf{y}(0)=\mathbf{y}_{0}$ to $\mathbf{y}(1)=\mathbf{z}$ in time $\tau:=N \tau_{0}$. I.e. $u \in U_{a d}$ and the corresponding solution $\mathbf{y}$ of the wave equation (2.2) with $\tau=N \tau_{0}$ satisfies $\mathbf{y}(0)=\mathbf{y}_{0}, \mathbf{y}(1)=\mathbf{z}$.

Proof. We first prove that for $N>0$ satisfying (2.7) there exists a control $u$ with $\|u\|_{L^{\infty}\left(I ; L^{2}(\omega)\right)} \leq \frac{\gamma}{2}$, driving the state $\mathbf{y}$ from $\mathbf{y}_{0}$ to $\mathbf{y}(1)=0$ in time $\tau=N \tau_{0}$.

We begin by proving an upper bound for the observability constant $c\left(N \tau_{0}\right)$ following an idea from [18], Theorem 3.1. Let $\mathbf{p}$ be an arbitrary solution of the adjoint equation (2.4) for $\tau:=N \tau_{0}$. For $j=1, \ldots, N$ let us define, in decreasing order, the functions $\mathbf{p}^{j}$ as the solution of

$$
-\left(\mathbf{p}^{j}\right)_{t}=\tau_{0} \mathbf{A}^{*} \mathbf{p}^{j} \quad \text { on } I, \quad \mathbf{p}^{j}(1)=\mathbf{p}^{j+1}(0)
$$

with $\mathbf{p}^{N+1}(0):=\mathbf{p}(1)$. Then for $t \in\left[(j-1) \tau_{0}, j \tau_{0}\right]$ we have $\mathbf{p}(t)=\mathbf{p}^{j}\left(t / \tau_{0}-(j-1)\right)$, in particular $\mathbf{p}(0)=\mathbf{p}^{1}(0)$. Due to energy conservation $\|\mathbf{p}(0)\|_{\mathbf{P}^{0}}=\|\mathbf{p}(1)\|_{\mathbf{P}^{0}}=\left\|\mathbf{p}^{j}(1)\right\|_{\mathbf{P}^{0}}$ holds.

Since the system is null-controllable for time $\tau_{0}$, it holds for all $j=1 \ldots N, c f .(2.5)$, that

$$
\left\|\mathbf{p}^{j}(1)\right\|_{\mathbf{P}^{0}}^{2} \leq c\left(\tau_{0}\right) \tau_{0}\left\|\mathbf{B}^{*} \mathbf{p}^{j}\right\|_{L^{2}\left(I ; L^{2}(\omega)\right)}^{2} .
$$

Summing this inequality for $j=1 \ldots N$, and using the relation between $\mathbf{p}$ and $\mathbf{p}^{j}$, we obtain

$$
\begin{aligned}
N\|\mathbf{p}(1)\|_{\mathbf{P}^{0}}^{2}=\sum_{j=1}^{N}\left\|\mathbf{p}^{j}(1)\right\|_{\mathbf{P}^{0}}^{2} & \leq c\left(\tau_{0}\right) \tau_{0} \sum_{j=1}^{N}\left\|\mathbf{B}^{*} \mathbf{p}^{j}\right\|_{L^{2}\left(I ; L^{2}(\omega)\right)}^{2} \\
& =c\left(\tau_{0}\right) \tau_{0} N\left\|\mathbf{B}^{*} \mathbf{p}\right\|_{L^{2}\left(I ; L^{2}(\omega)\right)}^{2},
\end{aligned}
$$


which proves

$$
\|\mathbf{p}(1)\|_{\mathbf{P}^{0}}^{2} \leq c\left(\tau_{0}\right) \tau_{0}\left\|\mathbf{B}^{*} \mathbf{p}\right\|_{L^{2}\left(I ; L^{2}(\omega)\right)}^{2} .
$$

This inequality implies an upper bound $c\left(N \tau_{0}\right) \leq N^{-1} c\left(\tau_{0}\right)$ for the observability constant $c\left(N \tau_{0}\right)$.

Now, let us turn to establish (2.6). By the properties of the adjoint wave equation, we have

$$
\begin{aligned}
& \|\mathbf{p}(1)\|_{\mathbf{P}^{0}}^{2} \leq c\left(\tau_{0}\right) \tau_{0}\left\|\mathbf{B}^{*} \mathbf{p}\right\|_{L^{2}\left(I ; L^{2}(\omega)\right)}^{2} \\
& \leq c\left(\tau_{0}\right) \tau_{0}\left\|\mathbf{B}^{*} \mathbf{p}\right\|_{L^{1}\left(I ; L^{2}(\omega)\right)}\left\|\mathbf{B}^{*} \mathbf{p}\right\|_{L^{\infty}\left(I ; L^{2}(\omega)\right)} \\
& \leq c\left(\tau_{0}\right) \tau_{0}\left\|\mathbf{B}^{*} \mathbf{p}\right\|_{L^{1}\left(I ; L^{2}(\omega)\right)}\left\|\mathbf{B}^{*}\right\|_{\mathcal{L}\left(\mathbf{P}^{0}, L^{2}(\omega)\right)}\|\mathbf{p}(1)\|_{\mathbf{P}^{0}} .
\end{aligned}
$$

Since $\left\|\mathbf{B}^{*}\right\|_{\mathcal{L}\left(\mathbf{P}^{0}, L^{2}(\omega)\right)} \leq 1$, we find

$$
\|\mathbf{p}(1)\|_{\mathbf{P}^{0}} \leq c\left(\tau_{0}\right) \tau_{0}\left\|\mathbf{B}^{*} \mathbf{p}\right\|_{L^{1}\left(I ; L^{2}(\omega)\right)} .
$$

Using (2.8) and conservation of energy, we obtain

$$
\begin{aligned}
\left\langle\mathbf{p}(0), \mathbf{y}_{0}\right\rangle_{\mathbf{P}^{0}, \mathbf{Y}^{1}}+\frac{\gamma}{2} N \tau_{0}\left\|\mathbf{B}^{*} \mathbf{p}\right\|_{L^{1}\left(I ; L^{2}(\omega)\right)} & \geq-\|\mathbf{p}(0)\|_{\mathbf{P}^{0}}\left\|\mathbf{y}_{0}\right\|_{\mathbf{Y}^{1}}+\frac{\gamma N}{2 c\left(\tau_{0}\right)}\|\mathbf{p}(1)\|_{\mathbf{P}^{0}} \\
& =\left(\frac{\gamma N}{2 c\left(\tau_{0}\right)}-\left\|\mathbf{y}_{0}\right\|_{\mathbf{Y}^{1}}\right)\|\mathbf{p}(1)\|_{\mathbf{P}^{0}} \geq 0
\end{aligned}
$$

where we used assumption (2.7). Hence, by the constrained controllability results $[1,17], c f .(2.6)$, there exists a control $u_{1}$ with $\left\|u_{1}\right\|_{L^{\infty}\left(I ; L^{2}(\omega)\right)} \leq \frac{\gamma}{2}$ that steers the system from $\mathbf{y}_{0}$ to $\mathbf{y}(1)=0$ in time $\tau=N \tau_{0}$. Similarly, one proves existence of a control $u_{2}$ with $\left\|u_{2}\right\|_{L^{\infty}\left(I ; L^{2}(\omega)\right)} \leq \frac{\gamma}{2}$ that steers the system from the initial state $\mathbf{y}(0)=0$ to $\mathbf{y}(1)=\mathbf{z}$ in time $\tau=N \tau_{0}$. Due to linearity of the wave equation, the control $u_{1}+u_{2}$ has the claimed properties.

Proposition 2.3 establishes the existence of feasible controls, from which existence of time-optimal controls easily follows. We summarize the above discussion as a theorem that gives a sufficient condition for the existence of a solution to $(\mathrm{P})$.

Theorem 2.4. Let $\mathbf{y}_{0}, \mathbf{z} \in \mathbf{Y}^{1}$ be given. If there is $\tau_{0}>0$ such that the wave-equation is null-controllable, i.e. (2.5) holds, then the time-optimal control problem admits a solution.

Proof. Due to Proposition 2.3 there exists a feasible control of problem (P). Existence of solution follows by standard subsequential limit arguments. See also [18], Theorem 4.2.

For constrained problems the notion of bang-bang controls is frequently of importance. Here we call a control $\tilde{u}$ bang-bang if $\|\tilde{u}(t)\|_{L^{2}}=\gamma$ for almost all $t \in I$. For $(\mathrm{P})$ it is well-known that in the case $\omega=\Omega$ all time-optimal controls are bang-bang [7], Theorem 6.12.3, page 304, and that $\|\tilde{u}(t)\|_{L^{2}}$ assumes the value $\gamma$ for all but finitely many $t \in I$. In this context also the maximum principle was established in [7], but the transversality condition was not addressed. To the best of our knowledge, it is an open problem under which conditions time-optimal controls are bang-bang when $\omega \neq \Omega$.

\section{A FAMily of REgUlarized PROBLEMS}

In this section we discuss a family of regularized problems that is obtained by penalization. Their optimality condition is derived and convergence of the primal variables is proven. There are several motivations to investigate such a regularization. Besides its intrinsic merit, the regularized formulation lends itself to obtain a first order optimality system and it is an appropriate starting point to derive numerical methods for the time optimal problem $(\mathrm{P})$. The regularization that we use consists in employing a cost term for the control and the realization of the terminal constraint as a penalty term. We note, that the control cost term may be quite natural 
in applications and need not necessarily be viewed as a regularization term. The use of the terminal penalty is further commented on in Remark 4.8 below. Smooth and non-smooth, but exact, penalization techniques of the terminal constraints were also employed in [8] for norm-optimal control problems. There for spatial dimension one, convergence rates with respect to the penalty parameter are investigated.

For $\varepsilon>0$ we consider

$$
\left\{\begin{array}{l}
\min J_{\varepsilon}(\tau, u)=\tau\left(1+\frac{\varepsilon}{2}\|u\|_{L^{2}\left(I ; L^{2}(\omega)\right)}^{2}\right)+\frac{1}{2 \varepsilon}\|\mathbf{y}(1)-\mathbf{z}\|_{\mathbf{Y}^{0}}^{2} \\
\text { subject to } \tau \geq 0 \text { and } \\
\mathbf{y}_{t}=\tau \mathbf{A} \mathbf{y}+\tau \mathbf{B} u, \text { on }(0,1] \\
\mathbf{y}(0)=\mathbf{y}_{0}, \\
u \in U_{a d}
\end{array}\right.
$$

Here and below the norm on $\mathbf{Y}^{0}=L^{2}(\Omega) \times H^{-1}(\Omega)$ is chosen to be

$$
\|\mathbf{v}\|_{\mathbf{Y}^{0}}^{2}=\left\|v_{1}\right\|_{L^{2}(\Omega)}^{2}+\left((-\Delta)^{-1} v_{2}, v_{2}\right)_{L^{2}(\Omega)},
$$

where $w=(-\Delta)^{-1} v_{2}$ is the solution of

$$
-\Delta w=v_{2} \text { in } \Omega, \quad w=0 \text { on } \Gamma .
$$

Problem $\left(P_{\varepsilon}\right)$ can be investigated without controllability assumption, but to guarantee convergence of its solution to a solution of $(\mathrm{P})$, it is, of course, required to assume that $(\mathrm{P})$ admits a solution. We therefore assume throughout the remainder of this paper:

$$
\text { Problem (P) admits a solution. }
$$

We have existence of solutions of $\left(P_{\varepsilon}\right)$ under our standing assumptions on the problem data.

Proposition 3.1. Problem $\left(P_{\varepsilon}\right)$ admits a solution.

Proof. Let $\left(\tau_{n}, y_{n}, u_{n}\right)$ denote a minimizing sequence. Since $J_{\varepsilon}$ is bounded from below, the sequence $\tau_{n}$ is bounded, and it therefore admits an accumulation point $\tilde{\tau}$. Since $u_{n} \in U_{a d}$ for all $n$, the sequence $\left\{u_{n}\right\}$ is bounded in $L^{\infty}\left(I ; L^{2}(\Omega)\right)$. By Theorem 2.1 , the sequence $\mathbf{y}_{n}$ is bounded in $C\left(\bar{I} ; \mathbf{Y}^{1}\right) \cap C^{1}\left(\bar{I} ; \mathbf{Y}^{0}\right)$. Choosing a weakly converging subsequence of $\left\{u_{n}\right\}$ in $L^{2}\left(I ; L^{2}(\omega)\right)$ and of $y_{n}$ in $L^{2}\left(I ; \mathbf{Y}^{1}\right) \cap H^{1}\left(I ; \mathbf{Y}^{0}\right)$ there exists $(\tilde{\tau}, \tilde{y}, \tilde{u})$ such that we can pass to the weak subsequential limit in

$$
\left\{\begin{array}{l}
\left(\mathbf{y}_{n}\right)_{t}=\tau_{n}\left(\mathbf{A} \mathbf{y}_{n}+\mathbf{B} u_{n}\right) \\
\mathbf{y}_{n}(0)=\mathbf{y}_{0}
\end{array}\right.
$$

to find that

$$
\begin{aligned}
& \tilde{\mathbf{y}}_{t}=\tilde{\tau}(\mathbf{A} \tilde{\mathbf{y}}+\mathbf{B} \tilde{u}) \\
& \tilde{\mathbf{y}}(0)=\mathbf{y}_{0} .
\end{aligned}
$$

Weak lower semi-continuity of $J_{\varepsilon}$ implies that $(\tilde{\tau}, \tilde{u})$ is a solution to $\left(P_{\varepsilon}\right)$.

In the sequel, $\left(\tau_{\varepsilon}, \mathbf{y}_{\varepsilon}, u_{\varepsilon}\right)$ denotes a solution of the penalized problem $\left(P_{\varepsilon}\right)$ for $\varepsilon>0$.

Theorem 3.2. Assume that (H1) holds and let $\left\{\left(\tau_{\varepsilon}, \mathbf{y}_{\varepsilon}, u_{\varepsilon}\right)\right\}_{\varepsilon>0}$ denote a family of solutions of $\left(P_{\varepsilon}\right)$. Then we have that

$$
\tau_{\varepsilon} \rightarrow \tau^{*}, \text { for } \varepsilon \rightarrow 0^{+}
$$


and $\left(\mathbf{y}_{\varepsilon}, u_{\varepsilon}\right)$ is uniformly bounded in $\left(C\left(\bar{I} ; \mathbf{Y}^{1}\right) \cap H^{1}\left(I ; \mathbf{Y}^{0}\right)\right) \times L^{\infty}\left(I ; L^{2}(\omega)\right)$. Moreover, for each weakly-star converging subsequence $\left\{\left(\mathbf{y}_{\varepsilon_{n}}, u_{\varepsilon_{n}}\right)\right\}$ with

$$
\mathbf{y}_{\varepsilon_{n}} \rightarrow^{*} \tilde{\mathbf{y}} \text { in } L^{\infty}\left(I ; \mathbf{Y}^{1}\right) \cap H^{1}\left(I ; \mathbf{Y}^{0}\right), \quad u_{\varepsilon_{n}} \rightarrow^{*} \tilde{u} \text { in } L^{\infty}\left(I ; L^{2}(\omega)\right),
$$

the limit $(\tilde{\mathbf{y}}, \tilde{u})$ is a solution of the original time-optimal control problem $(\mathrm{P})$.

If $\tilde{u}$ is bang-bang, then the convergence $\left(\mathbf{y}_{\varepsilon_{n}}, u_{\varepsilon_{n}}\right) \rightarrow(\tilde{\mathbf{y}}, \tilde{u})$ is strong in $\left(C\left(\bar{I} ; \mathbf{Y}^{1}\right) \cap H^{1}\left(I ; \mathbf{Y}^{0}\right)\right) \times L^{2}\left(I ; L^{2}(\omega)\right)$.

Proof. Let $\left(\tau^{*}, \mathbf{y}^{*}, u^{*}\right)$ denote a solution of $(\mathrm{P})$. Since it is feasible for the penalized problem we have

$$
\tau_{\varepsilon}\left(1+\frac{\varepsilon}{2}\left\|u_{\varepsilon}\right\|_{L^{2}\left(I ; L^{2}(\omega)\right)}^{2}\right)+\frac{1}{2 \varepsilon}\left\|\mathbf{y}_{\varepsilon}(1)-\mathbf{z}\right\|_{\mathbf{Y}^{0}}^{2} \leq \tau^{*}\left(1+\frac{\varepsilon}{2}\left\|u^{*}\right\|_{L^{2}\left(I ; L^{2}(\omega)\right)}^{2}\right) .
$$

This implies that $\limsup _{\varepsilon>0} \tau_{\varepsilon} \leq \tau^{*}$ and hence $\left\{\tau_{\varepsilon}: 0<\varepsilon<M\right\}$ is bounded for each $M \in(0, \infty)$.

Due to the control constraints and Theorem 2.1, the set $\left\{\mathbf{y}_{\varepsilon}, u_{\varepsilon}\right\}_{\varepsilon \in(0, M]}$ is bounded in

$$
\left(C\left(\bar{I} ; \mathbf{Y}^{1}\right) \cap H^{1}\left(I ; \mathbf{Y}^{0}\right)\right) \times L^{\infty}\left(I ; L^{2}(\omega)\right) .
$$

Let us choose a subsequence $\varepsilon_{n} \rightarrow 0$ such that $\tau_{\varepsilon_{n}} \rightarrow \tilde{\tau}, u_{\varepsilon_{n}} \rightarrow^{*} \tilde{u}$ in $L^{\infty}\left(I ; L^{2}(\omega)\right), \mathbf{y}_{\varepsilon_{n}} \rightarrow^{*} \tilde{\mathbf{y}}$ in $L^{\infty}\left(I ; \mathbf{Y}^{1}\right) \cap$ $H^{1}\left(I ; \mathbf{Y}^{0}\right)$ as $n \rightarrow \infty$. Arzela-Ascoli's theorem and the compact embedding $\mathbf{Y}^{1}$ in $\mathbf{Y}^{0}$ imply that we can choose $\varepsilon_{n}$ such that $\mathbf{y}_{\varepsilon_{n}} \rightarrow \tilde{\mathbf{y}}$ in $C\left(\bar{I} ; \mathbf{Y}^{0}\right)$ as $\varepsilon_{n} \rightarrow 0$. By $(3.1)$

$$
\mathbf{y}_{\varepsilon}(1) \rightarrow \mathbf{z} \text { in } \mathbf{Y}^{1}
$$

It further follows that $\tilde{\mathbf{y}}$ solves

$$
\begin{aligned}
\tilde{\mathbf{y}}_{t} & =\tilde{\tau} \mathbf{A} \tilde{\mathbf{y}}+\tilde{\tau} \mathbf{B} \tilde{u}, \\
\tilde{\mathbf{y}}(0) & =\mathbf{y}_{0}, \tilde{\mathbf{y}}(1)=\mathbf{z} .
\end{aligned}
$$

Since $U_{a d}$ is weakly closed, we have that $\tilde{u} \in U_{a d}$. Hence, $(\tilde{\tau}, \tilde{\mathbf{y}}, \tilde{u})$ is feasible for the time-optimal control problem with $\tilde{\tau} \leq \tau^{*}$. Since $\tau^{*}$ was the minimal time, $\tilde{\tau}=\tau^{*}$ and $(\tilde{\tau}, \tilde{\mathbf{y}}, \tilde{u})$ is a solution of the time-optimal control problem. The minimal time $\tau^{*}$ is unique, and consequently the whole family $\tau_{\varepsilon}$ converges to $\tau^{*}$ as $\varepsilon \rightarrow 0^{+}$.

If $\tilde{u}$ is bang-bang, then we have by feasibility of $u_{\varepsilon_{n}}$ and weakly lower-semicontinuity of norms

$$
\|\tilde{u}\|_{L^{2}\left(I ; L^{2}(\omega)\right)}=\gamma \geq \limsup \left\|u_{\varepsilon_{n}}\right\|_{L^{2}\left(I ; L^{2}(\omega)\right)} \geq \liminf \left\|u_{\varepsilon_{n}}\right\|_{L^{2}\left(I ; L^{2}(\omega)\right)} \geq\|\tilde{u}\|_{L^{2}\left(I ; L^{2}(\omega)\right)} .
$$

This implies norm convergence $\lim _{n \rightarrow \infty}\left\|u_{\varepsilon_{n}}\right\|_{L^{2}\left(I ; L^{2}(\omega)\right)}=\|\tilde{u}\|_{L^{2}\left(I ; L^{2}(\omega)\right)}$ and the strong convergence, as stated, is obtained.

As already discussed in Section 2, the assumption on the bang-bang nature of all time-optimal controls holds for the case $\omega=\Omega$.

Corollary 3.3. Under the assumptions of the previous theorem, we have

$$
\left\|\mathbf{y}_{\varepsilon}(1)-\mathbf{z}\right\|_{\mathbf{Y}^{0}}^{2} \leq \varepsilon\left(2\left|\tau_{\varepsilon}-\tau^{*}\right|+O(\varepsilon)\right)
$$

for $\varepsilon \rightarrow 0^{+}$.

Proof. Inequality (3.1) implies

$$
\left\|\mathbf{y}_{\varepsilon}(1)-\mathbf{z}\right\|_{\mathbf{Y}^{0}}^{2} \leq 2 \varepsilon\left|\tau^{*}-\tau_{\varepsilon}\right|+\varepsilon^{2}\left(\tau^{*}\left\|u^{*}\right\|_{L^{2}\left(I ; L^{2}(\omega)\right)}^{2}-\tau_{\varepsilon}\left\|u_{\varepsilon}\right\|_{L^{2}\left(I ; L^{2}(\omega)\right)}^{2}\right) .
$$

Since the set of admissible controls is bounded, the claim follows immediately. 
Remark 3.4. In numerical practice we find cases where $\left|\tau_{\varepsilon}-\tau^{*}\right|=O(\varepsilon)$. Then $\left\|\mathbf{y}_{\varepsilon}(1)-\mathbf{z}\right\|_{\mathbf{Y}^{0}}^{2}=O\left(\varepsilon^{2}\right)$.

First order necessary optimality conditions for $\left(P_{\varepsilon}\right)$ are derived next. To employ the method of transposition the adjoint state $\mathbf{p}_{\varepsilon}$ is defined as the solution of

$$
-\mathbf{p}_{t}=\tau \mathbf{A}^{*} \mathbf{p}
$$

with terminal condition

$$
\mathbf{p}(1)=\frac{1}{\varepsilon}\left(\begin{array}{r}
\mathbf{y}_{\varepsilon, 1}(1)-z_{1} \\
(-\Delta)^{-1}\left(\mathbf{y}_{\varepsilon, 2}(1)-z_{2}\right)
\end{array}\right)
$$

We note that

$$
\mathbf{p}_{\varepsilon}(1) \in \mathbf{P}^{2}
$$

and hence by Theorem 2.1 we have the following regularity result for $\mathbf{p}_{\varepsilon}$.

Corollary 3.5. Let $\mathbf{y}_{\varepsilon} \in C\left(\bar{I} ; \mathbf{Y}^{1}\right)$. Then (3.2)-(3.3) admits a unique solution $\mathbf{p}_{\varepsilon} \in C\left(\bar{I} ; \mathbf{P}^{2}\right) \cap C^{1}\left(\bar{I} ; \mathbf{P}^{1}\right)$.

After these preliminaries we obtain the first order necessary condition for $\left(P_{\varepsilon}\right)$.

Theorem 3.6. Let $\left(\tau_{\varepsilon}, \mathbf{y}_{\varepsilon}, u_{\varepsilon}\right)$ be a local solution of $\left(P_{\varepsilon}\right)$. Then there exists $\mathbf{p}_{\varepsilon} \in C\left(\bar{I} ; \mathbf{P}^{2}\right) \cap C^{1}\left(\bar{I} ; \mathbf{P}^{1}\right)$ such that the following optimality system holds:

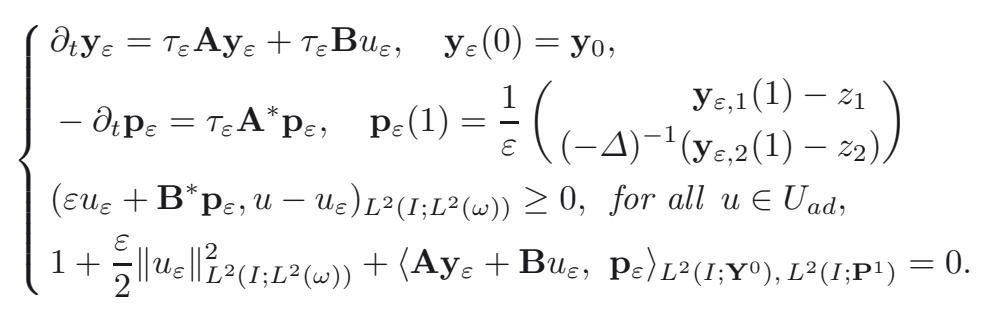

The optimal control $u_{\varepsilon}$ has the additional regularity

$$
u_{\varepsilon} \in C\left(\bar{I} ; L^{2}(\omega)\right) \text { and } \partial_{t} u_{\varepsilon} \in L^{\infty}\left(I ; L^{2}(\omega)\right)
$$

Moreover, if $\mathbf{y}_{0} \in \mathbf{Y}^{2}$ then

$$
\mathbf{y}_{\varepsilon} \in C\left(\bar{I} ; \mathbf{Y}^{2}\right) \cap C^{1}\left(\bar{I} ; \mathbf{Y}^{1}\right) .
$$

We refer to the four assertions in (3.4) as primal- and adjoint equations, optimality- and transversality condition. We note that $\mathbf{B}^{*} \mathbf{p}_{\varepsilon}$ is the restriction operator to $\omega$ given by $\left(\mathbf{B}^{*} \mathbf{p}_{\varepsilon}\right)(x)=\mathbf{p}_{\varepsilon, 2}(x)$ for $x \in \omega$.

Proof. Let us take $u \in U_{a d}$ and set $h=u-u_{\varepsilon}$. Further let $\tilde{\mathbf{y}} \in C\left(\bar{I} ; \mathbf{Y}^{1}\right)$ denote the solution to the sensitivity equation with respect to $u$ of the primal equation, i.e. to

$$
\begin{aligned}
\partial_{t} \tilde{\mathbf{y}} & =\tau_{\varepsilon}(\mathbf{A} \tilde{\mathbf{y}}+\mathbf{B} h) \\
\tilde{\mathbf{y}}(0) & =0 .
\end{aligned}
$$

Then the directional derivative $\partial_{u} J_{\varepsilon}\left(\tau_{\varepsilon}, u_{\varepsilon}\right) h$ at $\left(\tau_{\varepsilon}, u_{\varepsilon}\right)$ in the admissible directions $h$ satisfies $\partial_{u} J_{\varepsilon}\left(\tau_{\varepsilon}, u_{\varepsilon}\right) h \geq 0$ and it is given by

$$
\begin{aligned}
\partial_{u} J_{\varepsilon}\left(\tau_{\varepsilon}, u_{\varepsilon}\right) h & =\varepsilon \tau_{\varepsilon}\left(u_{\varepsilon}, h\right)_{L^{2}\left(I ; L^{2}(\omega)\right)}+\left(\tilde{\mathbf{y}}(1), \mathbf{p}_{\varepsilon}(1)\right)_{L^{2}(\Omega)} \\
& =\varepsilon \tau_{\varepsilon}\left(u_{\varepsilon}, h\right)_{L^{2}\left(I ; L^{2}(\omega)\right)}+\int_{0}^{1} \frac{\mathrm{d}}{\mathrm{d} t}\left(\tilde{\mathbf{y}}(t), \mathbf{p}_{\varepsilon}(t)\right)_{L^{2}(\Omega)} \mathrm{d} t \\
& =\varepsilon \tau_{\varepsilon}\left(u_{\varepsilon}, h\right)_{L^{2}\left(I ; L^{2}(\omega)\right)}+\tau_{\varepsilon} \int_{0}^{1}\left(\left\langle\mathbf{A} \tilde{\mathbf{y}}+\mathbf{B} h, \mathbf{p}_{\varepsilon}\right\rangle-\left\langle\tilde{\mathbf{y}}, \mathbf{A}^{*} \mathbf{p}_{\varepsilon}\right\rangle\right) \mathrm{d} t \\
& =\tau_{\varepsilon}\left(\varepsilon u_{\varepsilon}+\mathbf{B}^{*} \mathbf{p}_{\varepsilon}, h\right)_{L^{2}\left(I ; L^{2}(\omega)\right)} \geq 0
\end{aligned}
$$


proves the optimality condition. Similarly let $\hat{\mathbf{y}}$ denote the solution of the sensitivity equation of the primal equation with respect to $\tau>0$, i.e. of

$$
\begin{aligned}
\partial_{t} \hat{\mathbf{y}} & =\tau_{\varepsilon} \mathbf{A} \hat{\mathbf{y}}+\mathbf{A y}_{\varepsilon}+\mathbf{B} u_{\varepsilon} \\
\hat{\mathbf{y}}(0) & =0 .
\end{aligned}
$$

Then

$$
\begin{aligned}
0 & =\partial_{\tau} J_{\varepsilon}\left(\tau_{\varepsilon}, u_{\varepsilon}\right)=1+\frac{\varepsilon}{2}\left\|u_{\varepsilon}\right\|_{L^{2}\left(I ; L^{2}(\omega)\right)}^{2}+\frac{1}{\varepsilon}\left\langle\mathbf{y}_{\varepsilon}(1)-\mathbf{z}, \hat{\mathbf{y}}(1)\right\rangle_{\mathbf{Y}^{0}} \\
& =1+\frac{\varepsilon}{2}\left\|u_{\varepsilon}\right\|_{L^{2}\left(I ; L^{2}(\omega)\right)}^{2}+\left(\hat{\mathbf{y}}(1), \mathbf{p}_{\varepsilon}(1)\right)_{L^{2}(\Omega)} \\
& =1+\frac{\varepsilon}{2}\left\|u_{\varepsilon}\right\|_{L^{2}\left(I ; L^{2}(\omega)\right)}^{2}+\int_{0}^{1} \frac{\mathrm{d}}{\mathrm{d} t}\left(\hat{\mathbf{y}}(t), \mathbf{p}_{\varepsilon}(t)\right)_{L^{2}(\Omega)} \mathrm{d} t \\
& =1+\frac{\varepsilon}{2}\left\|u_{\varepsilon}\right\|_{L^{2}\left(I ; L^{2}(\omega)\right)}^{2}+\int_{0}^{1}\left(\left\langle\tau_{\varepsilon} \mathbf{A} \hat{\mathbf{y}}(t)+\mathbf{A y}_{\varepsilon}(t)+\mathbf{B} u_{\varepsilon}(t), \mathbf{p}_{\varepsilon}(t)\right\rangle_{\mathbf{Y}^{0}, \mathbf{P}^{1}}-\left(\hat{\mathbf{y}}(t), \tau_{\varepsilon} \mathbf{A}^{*} \mathbf{p}_{\varepsilon}(t)\right)\right) \mathrm{d} t \\
& =1+\frac{\varepsilon}{2}\left\|u_{\varepsilon}\right\|_{L^{2}\left(I ; L^{2}(\omega)\right)}^{2}+\int_{0}^{1}\left\langle\mathbf{A y}_{\varepsilon}(t)+\mathbf{B} u_{\varepsilon}(t), \mathbf{p}_{\varepsilon}(t)\right\rangle_{\mathbf{Y}^{0}, \mathbf{P}^{1}} \mathrm{~d} t
\end{aligned}
$$

which proves the transversality condition. The third condition in (3.4) is equivalent to

$$
u_{\varepsilon}(t)=\mathrm{P}_{U}\left(-\frac{1}{\varepsilon} \chi_{\omega} \mathbf{p}_{\varepsilon, 2}(t)\right) \quad \text { for almost all } t \in I,
$$

which implies that, after modifying $u_{\varepsilon}$ on a set of measure zero in $I$, we have $u_{\varepsilon} \in C\left(\bar{I} ; L^{2}(\omega)\right)$, hence the point-wise representation holds everywhere on the closed interval. Here $\mathrm{P}_{U}$ denotes the canonical projection in $L^{2}(\omega)$ onto $U$. With Lemma 3.7 below, we conclude $\partial_{t} u_{\varepsilon} \in L^{\infty}\left(I ; L^{2}(\omega)\right)$, which in turn gives the higher regularity $\mathbf{y}_{\varepsilon} \in C\left(\bar{I} ; \mathbf{Y}^{2}\right)$, under the additional requirement that $\mathbf{y}_{0} \in \mathbf{Y}^{2}$.

Lemma 3.7. Let $q \in C^{1}\left(\bar{I} ; L^{2}(\omega)\right)$ be given. Then $u$ defined by

$$
u(t)=\mathrm{P}_{U}(q(t))
$$

is in $C\left(\bar{I} ; L^{2}(\omega)\right)$ with $\partial_{t} u \in L^{\infty}\left(I ; L^{2}(\omega)\right)$ and

$$
\left\|\partial_{t} u\right\|_{L^{\infty}\left(I ; L^{2}(\omega)\right)} \leq 2\left\|\partial_{t} q\right\|_{L^{\infty}\left(I ; L^{2}(\omega)\right)} .
$$

Proof. The definition of $U$ implies that

$$
u(t)=\frac{q(t)}{\max \left(1,\|q(t)\|_{L^{2}(\omega)} / \gamma\right)},
$$

which proves that $u \in C^{0,1}\left(\bar{I} ; L^{2}(\omega)\right)$. Then the weak time derivative of $u$ satisfies

$$
\partial_{t} u(t)= \begin{cases}\partial_{t} q(t) & \|q(t)\|_{L^{2}(\omega)} \leq \gamma \\ \gamma \frac{\|q(t)\|_{L^{2}(\omega)}^{2} \partial_{t} q(t)-\left(q(t), \partial_{t} q(t)\right)_{L^{2}(\omega)} q(t)}{\|q(t)\|_{L^{2}(\omega)}^{3}} & \|q(t)\|_{L^{2}(\omega)}>\gamma .\end{cases}
$$

Due to the regularity of $q$ we have $\partial_{t} u \in L^{\infty}\left(I ; L^{2}(\omega)\right)$.

The following corollaries concern themselves with the pointwise transversality condition of the regularized problems which we express in term of the Hamiltonian

$$
\mathcal{H}(\mathbf{y}, u, \mathbf{p})=1+\frac{\varepsilon}{2}\|u\|_{L^{2}(\omega)}^{2}+\langle\mathbf{A y}+\mathbf{B} u, \mathbf{p}\rangle_{\mathbf{Y}^{0}, \mathbf{P}^{1}},
$$

for $(\mathbf{y}, u, \mathbf{p}) \in \mathbf{Y}^{1} \times L^{2}(\omega) \times \mathbf{P}^{1}$. 
Corollary 3.8 (pointwise transversality). Let $\mathbf{y}_{0} \in \mathbf{Y}^{2}$ and let $\left(\tau_{\varepsilon}, \mathbf{y}_{\varepsilon}, u_{\varepsilon}\right)$ be a local solution of $\left(P_{\varepsilon}\right)$ with associated adjoint state $\mathbf{p}_{\varepsilon}$. Then we have

$$
\mathcal{H}\left(\mathbf{y}_{\varepsilon}(t), u_{\varepsilon}(t), \mathbf{p}_{\varepsilon}(t)\right)=0 \text { for all } t \in \bar{I} .
$$

Proof. Due to the regularity of $\mathbf{y}_{\varepsilon}$ and $\mathbf{p}_{\varepsilon}$, we have $\mathbf{y}_{\varepsilon} \in C^{1}\left(\bar{I} ; \mathbf{Y}^{1}\right), \mathbf{A y}_{\varepsilon} \in C^{1}\left(\bar{I} ; \mathbf{Y}^{0}\right)=C^{1}\left(\bar{I} ;\left(\mathbf{P}^{1}\right)^{*}\right)$, and $\mathbf{p}_{\varepsilon} \in C^{1}\left(\bar{I} ; \mathbf{P}^{1}\right)$. Hence the mapping $t \mapsto\left\langle\mathbf{A y}_{\varepsilon}(t), \mathbf{p}_{\varepsilon}(t)\right\rangle_{\left(\mathbf{P}^{1}\right)^{*}, \mathbf{P}^{1}}$ is continuously differentiable. Additionally, the optimality conditions imply

$$
\left(\varepsilon u_{\varepsilon}(t)+\mathbf{B}^{*} \mathbf{p}_{\varepsilon}(t), \partial_{t} u_{\varepsilon}(t)\right)_{L^{2}(\omega)}=0
$$

for almost all $t \in I$. Let us introduce the function

$$
g(t)=\frac{\varepsilon}{2}\left\|u_{\varepsilon}(t)\right\|_{L^{2}(\omega)}^{2}+\left\langle\mathbf{A y}_{\varepsilon}(t)+\mathbf{B} u_{\varepsilon}(t), \mathbf{p}_{\varepsilon}(t)\right\rangle_{\left(\mathbf{P}^{1}\right)^{*}, \mathbf{P}^{1}}
$$

Differentiating w.r.t. $t$, we obtain for almost all $t \in I$

$$
\partial_{t} g(t)=\left(\varepsilon u_{\varepsilon}(t)+\mathbf{B}^{*} \mathbf{p}_{\varepsilon}(t), \partial_{t} u_{\varepsilon}(t)\right)_{L^{2}(\omega)}+\left\langle\mathbf{A} \mathbf{y}_{\varepsilon}(t)+\mathbf{B} u_{\varepsilon}(t), \partial_{t} \mathbf{p}_{\varepsilon}(t)\right\rangle_{\left(\mathbf{P}^{1}\right)^{*}, \mathbf{P}^{1}}+\left\langle\mathbf{A} \partial_{t} \mathbf{y}_{\varepsilon}(t), \mathbf{p}_{\varepsilon}(t)\right\rangle_{\left(\mathbf{P}^{1}\right)^{*}, \mathbf{P}^{1}},
$$

which proves that $\partial_{t} g \in L^{2}(I)$. Taking into account (3.5), we obtain

$$
\begin{aligned}
\partial_{t} g(t) & =\left\langle\mathbf{A y}_{\varepsilon}(t)+\mathbf{B} u_{\varepsilon}(t), \partial_{t} \mathbf{p}_{\varepsilon}(t)\right\rangle_{\left(\mathbf{P}^{1}\right)^{*}, \mathbf{P}^{1}}+\left\langle\mathbf{A} \partial_{t} \mathbf{y}_{\varepsilon}(t), \mathbf{p}_{\varepsilon}(t)\right\rangle_{\left(\mathbf{P}^{1}\right)^{*}, \mathbf{P}^{1}} \\
& =\frac{1}{\tau_{\varepsilon}}\left(\left\langle\partial_{t} \mathbf{y}_{\varepsilon}(t), \partial_{t} \mathbf{p}_{\varepsilon}(t)\right\rangle_{\left(\mathbf{P}^{1}\right)^{*}, \mathbf{P}^{1}}-\left\langle\partial_{t} \mathbf{y}_{\varepsilon}(t), \partial_{t} \mathbf{p}_{\varepsilon}(t)\right\rangle_{\left(\mathbf{P}^{1}\right)^{*}, \mathbf{P}^{1}}\right)=0 .
\end{aligned}
$$

Hence we find $\partial_{t} g(t)=0$ on $I$, and $g(t)=$ constant on $I$. The last equation in (3.4) implies that this constant equals -1 . This implies the claim since $\left(\mathbf{P}^{1}\right)^{*}=\mathbf{Y}^{0}$.

The transversality condition of Corollary 3.8 for $t=1$ can be simplified using the explicit expression for $\mathbf{p}_{\varepsilon}(1)$.

Corollary 3.9. Let $\mathbf{y}_{0} \in \mathbf{Y}^{2}$ and let $\left(\tau_{\varepsilon}, \mathbf{y}_{\varepsilon}, u_{\varepsilon}\right)$ be a local solution of $\left(P_{\varepsilon}\right)$ with associated adjoint state $\mathbf{p}_{\varepsilon}$. Then we have

$$
\left(\mathbf{A y}_{\varepsilon}(1), \mathbf{p}_{\varepsilon}(1)\right)_{L^{2}(\Omega)^{2}}=\left\langle\mathbf{z}, \mathbf{A}^{*} \mathbf{p}_{\varepsilon}(1)\right\rangle_{L^{2}(\Omega)^{2}} .
$$

If additionally $\mathbf{z} \in \mathbf{Y}^{2}$, then we obtain

$$
\left(\mathbf{A y}_{\varepsilon}(1), \mathbf{p}_{\varepsilon}(1)\right)_{L^{2}(\Omega)^{2}}=\left\langle\mathbf{A z}, \mathbf{p}_{\varepsilon}(1)\right\rangle_{\left(\mathbf{P}^{0}\right)^{*}, \mathbf{P}^{0}} .
$$

Proof. Using the definition of $\mathbf{p}_{\varepsilon}(1)$ and of the operator $\mathbf{A}$, we find

$$
\begin{aligned}
\left(\mathbf{A y}_{\varepsilon}(1), \mathbf{p}_{\varepsilon}(1)\right)_{L^{2}(\Omega)^{2}} & =\left(\mathbf{y}_{\varepsilon, 2}(1), \mathbf{p}_{\varepsilon, 1}(1)\right)_{L^{2}(\Omega)}+\left(\Delta \mathbf{y}_{\varepsilon, 1}(1), \mathbf{p}_{\varepsilon, 2}(1)\right)_{L^{2}(\Omega)} \\
& =\frac{1}{\varepsilon}\left(\mathbf{y}_{\varepsilon, 2}(1), \mathbf{y}_{\varepsilon, 1}(1)-z_{1}\right)_{L^{2}(\Omega)}-\frac{1}{\varepsilon}\left(\mathbf{y}_{\varepsilon, 1}(1), \mathbf{y}_{\varepsilon, 2}(1)-z_{2}\right)_{L^{2}(\Omega)} \\
& =-\frac{1}{\varepsilon}\left(\mathbf{y}_{\varepsilon, 2}(1), z_{1}\right)_{L^{2}(\Omega)}+\frac{1}{\varepsilon}\left(\mathbf{y}_{\varepsilon, 1}(1), z_{2}\right)_{L^{2}(\Omega)} \\
& =-\frac{1}{\varepsilon}\left(\mathbf{y}_{\varepsilon, 2}(1)-z_{2}, z_{1}\right)_{L^{2}(\Omega)}+\frac{1}{\varepsilon}\left(\mathbf{y}_{\varepsilon, 1}(1)-z_{1}, z_{2}\right)_{L^{2}(\Omega)} .
\end{aligned}
$$

Again by the definition of $\mathbf{p}_{\varepsilon}(1)$, we have

$$
\left(\mathbf{A y}_{\varepsilon}(1), \mathbf{p}_{\varepsilon}(1)\right)_{L^{2}(\Omega)^{2}}=\left\langle\Delta \mathbf{p}_{\varepsilon, 2}(1), z_{1}\right\rangle_{H^{-1}(\Omega), H_{0}^{1}(\Omega)}+\left(\mathbf{p}_{\varepsilon, 1}(1), z_{2}\right)_{L^{2}(\Omega)} .
$$

If $\mathbf{z} \in \mathbf{Y}^{2}$, then $\mathbf{A} \mathbf{z} \in \mathbf{Y}^{1}=\left(\mathbf{P}^{0}\right)^{*}$, which finishes the proof. 
Remark 3.10. The relationship between primal and adjoint variables as well as $\mathbf{z}$, expressed in equation (3.6) is remarkable in its own right. In fact, if the regularized problem $\left(P_{\varepsilon}\right)$ had been defined with the terminal conditions as constraint $\mathbf{y}(1)=\mathbf{z}$, rather than as penalty, then this would again result in (3.6). This issue will be further addressed in Remark 4.8 .

Combining Corollary 3.8 for $t=1$ and (3.6) we obtain a form of the transversality condition where the duality pairing depends on $\mathbf{z}$ rather the $\varepsilon$-dependent quantity $\mathbf{y}_{\varepsilon}$.

Corollary 3.11. Let the assumptions of the Corollary 3.8 be satisfied and assume that $\mathbf{z} \in \mathbf{Y}^{2}$. Then the transversality condition at $t=1$ is given by

$$
1+\frac{\varepsilon}{2}\left\|u_{\varepsilon}(1)\right\|_{L^{2}(\omega)}^{2}+\left\langle\mathbf{A z}+\mathbf{B} u_{\varepsilon}(1), \mathbf{p}_{\varepsilon}(1)\right\rangle_{\left(\mathbf{P}^{0}\right)^{*}, \mathbf{P}^{0}}=0 .
$$

\section{Optimality SYSTEM AND TRANSVERSALITY CONDITION}

Here we are concerned with the asymptotic behavior of (3.4) as $\varepsilon \rightarrow 0^{+}$. For the primal equation this was addressed in Theorem 3.2. We aim at obtaining a first order necessary optimality system for the original time optimal control problem. This will involve the primal and adjoint equations, the maximum principle involving the optimal control, and, the transversality condition, which, to the best of our knowledge has received little attention so far for time optimal control of the wave equation.

Throughout this section, we will impose (H1) and the regularity assumptions

$$
\mathbf{y}_{0}, \mathbf{z} \in \mathbf{Y}^{2},
$$

which were used in Corollary 3.11, and which will be referred to in the proofs of the section. We will further rely on the observability condition, which was already discussed in Section 2.2.

We start with two lemmata.

Lemma 4.1. For each $t \in \bar{I}$ we have

$$
\left(u_{\varepsilon}(t), \mathbf{B}^{*} \mathbf{p}_{\varepsilon}(t)\right)_{L^{2}(\omega)}=-\varepsilon\left\|u_{\varepsilon}(t)\right\|_{L^{2}(\omega)}^{2}-\gamma\left\|\varepsilon u_{\varepsilon}(t)+\mathbf{B}^{*} \mathbf{p}_{\varepsilon}(t)\right\|_{L^{2}(\omega)}
$$

and

$$
\left(u_{\varepsilon}, \mathbf{B}^{*} \mathbf{p}_{\varepsilon}\right)_{L^{2}\left(I ; L^{2}(\omega)\right)}=-\varepsilon\left\|u_{\varepsilon}\right\|_{L^{2}\left(I ; L^{2}(\omega)\right)}^{2}-\gamma\left\|\varepsilon u_{\varepsilon}+\mathbf{B}^{*} \mathbf{p}_{\varepsilon}\right\|_{L^{1}\left(I ; L^{2}(\omega)\right)} .
$$

Proof. In the inactive case $\frac{1}{\varepsilon}\left\|\mathbf{B}^{*} \mathbf{p}_{\varepsilon}(t)\right\|_{L^{2}(\omega)} \leq \gamma$, we obtain from (3.4) that $u_{\varepsilon}(t)=-\frac{1}{\varepsilon} \mathbf{B}^{*} \mathbf{p}_{\varepsilon}(t)$, and thus

$$
\left(u_{\varepsilon}(t), \mathbf{B}^{*} \mathbf{p}_{\varepsilon}(t)\right)_{L^{2}(\omega)}=-\frac{1}{\varepsilon}\left\|\mathbf{B}^{*} \mathbf{p}_{\varepsilon}(t)\right\|_{L^{2}(\omega)}^{2} \geq-\gamma\left\|\mathbf{B}^{*} \mathbf{p}_{\varepsilon}(t)\right\|_{L^{2}(\omega)} .
$$

Since $\left(u_{\varepsilon}(t), \mathbf{B}^{*} \mathbf{p}_{\varepsilon}(t)\right)_{L^{2}(\omega)}=-\varepsilon\left\|u_{\varepsilon}(t)\right\|_{L^{2}(\omega)}^{2}$ and $\varepsilon u_{\varepsilon}(t)+\mathbf{B}^{*} \mathbf{p}_{\varepsilon}(t)=0$, we find (4.1) for the inactive case.

In the active case $\frac{1}{\varepsilon}\left\|\mathbf{B}^{*} \mathbf{p}_{\varepsilon}(t)\right\|_{L^{2}(\omega)}>\gamma$, the optimality condition (3.4) implies $u_{\varepsilon}(t)=$ $-\frac{\gamma}{\left\|\mathbf{B}^{*} \mathbf{p}_{\varepsilon}(t)\right\|_{L^{2}(\omega)}} \mathbf{B}^{*} \mathbf{p}_{\varepsilon}(t)$. Moreover

$$
\begin{aligned}
\varepsilon\left\|u_{\varepsilon}(t)\right\|_{L^{2}(\omega)}^{2}+\gamma\left\|\varepsilon u_{\varepsilon}(t)+\mathbf{B}^{*} \mathbf{p}_{\varepsilon}(t)\right\|_{L^{2}(\omega)} & =\varepsilon \gamma^{2}+\gamma\left(1-\frac{\varepsilon \gamma}{\left\|\mathbf{B}^{*} \mathbf{p}_{\varepsilon}(t)\right\|_{L^{2}(\omega)}}\right)\left\|\mathbf{B}^{*} \mathbf{p}_{\varepsilon}(t)\right\|_{L^{2}(\omega)} \\
& =\gamma\left\|\mathbf{B}^{*} \mathbf{p}_{\varepsilon}(t)\right\|_{L^{2}(\omega)}=-\left(u_{\varepsilon}(t), \mathbf{B}^{*} \mathbf{p}_{\varepsilon}(t)\right)_{L^{2}(\omega)},
\end{aligned}
$$

which proves (4.1) for the active case. Equality (4.2) follows from (4.1).

The following lemma employs assumption (H2), which is assumed to hold throughout this section. 
Lemma 4.2. There exists $\bar{\varepsilon}>0$ and $\delta>0$ such that $\left\|\mathbf{p}_{\varepsilon}(1)\right\|_{\mathbf{P}^{0}} \geq \delta$ for all $\varepsilon \in(0, \bar{\varepsilon}]$,

Proof. If the claim was false, then there exists a sequence $\left\{\varepsilon_{n}\right\}_{n=1}^{\infty}$ with $\lim _{n \rightarrow \infty} \varepsilon_{n}=0$ and $\lim _{n \rightarrow \infty}\left\|\mathbf{p}_{\varepsilon_{n}}(1)\right\|_{\mathbf{P}^{0}}=0$. From (3.7) we have

$$
1+\frac{\varepsilon_{n}}{2}\left\|u_{\varepsilon_{n}}(1)\right\|_{L^{2}(\omega)}^{2}+\left(\Delta z_{1}, \mathbf{p}_{\varepsilon_{n}, 2}(1)\right)_{L^{2}(\Omega)}+\left\langle\mathbf{p}_{\varepsilon_{n}, 1}(1), z_{2}\right\rangle_{H^{-1}, H^{1}}+\left(\chi_{\omega} u_{\varepsilon_{n}}(1), \mathbf{p}_{\varepsilon_{n}, 2}(1)\right)_{L^{2}(\Omega)}=0
$$

Since by assumption $\mathbf{z} \in \mathbf{Y}^{2}$, all addends tend to zero except for the first one. This is impossible.

In the following theorems we consider, according to Theorem 3.2, a weakly-star convergent subsequence of $\left\{\left(\tau_{\varepsilon}, y_{\varepsilon}, u_{\varepsilon}\right)\right\}_{\varepsilon>0}$, denoted by the same symbol with

$$
\left(\tau_{\varepsilon}, \mathbf{y}_{\varepsilon}, u_{\varepsilon}\right) \rightarrow^{*}\left(\tau^{*}, \tilde{\mathbf{y}}, \tilde{u}\right) \text { in } \mathbb{R} \times\left(L^{\infty}\left(I ; \mathbf{Y}^{1}\right) \cap H^{1}\left(I ; \mathbf{Y}^{0}\right)\right) \times L^{2}\left(I ; L^{2}(\omega)\right),
$$

as $\varepsilon \rightarrow 0^{*}$, where $\left(\tau^{*}, \tilde{\mathbf{y}}, \tilde{u}\right)$ is a solution of $(\mathrm{P})$.

Convergence of the solutions of the regularized optimality system to a solution of the optimality system for $(\mathrm{P})$ will now be derived under essentially two different types of conditions. The first one is the bang-bang property of the control to $(\mathrm{P})$, and the second one a boundedness condition on $\left\{\frac{\mathbf{p}_{\varepsilon}(1)}{\left\|\mathbf{p}_{\varepsilon}(1)\right\|_{\mathbf{P}^{0}}}\right\}_{\varepsilon \in(0,1]}$ in $\mathbf{P}^{1}$.

Theorem 4.3. Suppose that $(2.1)$ is controllable for some $T<\tau^{*}$ and that $\tilde{u}$ is bang-bang. Then there exists a non-trivial $\tilde{\mathbf{p}} \in C\left(\overline{\bar{I}} ; \mathbf{P}^{0}\right)$ such that

$$
\left\{\begin{array}{l}
\tilde{\mathbf{y}}_{t}=\tau^{*} \mathbf{A} \tilde{\mathbf{y}}+\tau^{*} \mathbf{B} \tilde{u}, \tilde{\mathbf{y}}(0)=\mathbf{y}_{0}, \tilde{\mathbf{y}}(1)=\mathbf{z} \\
-\tilde{\mathbf{p}}_{t}=\tau^{*} \mathbf{A}^{*} \tilde{\mathbf{p}} \\
\left(\mathbf{B}^{*} \tilde{\mathbf{p}}, u-\tilde{u}\right)_{L^{2}\left(I ; L^{2}(\omega)\right)} \geq 0 \text { for all } u \in U_{a d}
\end{array}\right.
$$

If, moreover $\mathbf{B}^{*} \tilde{\mathbf{p}}(1) \neq 0$, then

(a) if $\left\{\mathbf{p}_{\varepsilon}(1)\right\}$ is bounded in $\mathbf{P}^{0}$, then

$$
1+\langle\mathbf{A z}+\mathbf{B} \tilde{u}(1), \tilde{\mathbf{p}}(1)\rangle_{\left(\mathbf{P}^{0}\right)^{*}, \mathbf{P}^{0}}=0 ;
$$

(b) if $\left\{\mathbf{p}_{\varepsilon}(1)\right\}$ is unbounded in $\mathbf{P}^{0}$, then $\mathbf{z} \neq 0$ and

$$
\langle\mathbf{A z}+\mathbf{B} \tilde{u}(1), \tilde{\mathbf{p}}(1)\rangle_{\left(\mathbf{P}^{0}\right)^{*}, \mathbf{P}^{0}}=0 .
$$

Proof. Let us assume at first that

$$
\left\{\mathbf{p}_{\varepsilon}(1)\right\} \text { is bounded } \mathbf{P}^{0} .
$$

Then $\left\{\mathbf{p}_{\varepsilon}\right\}$ is bounded in $C\left(\bar{I} ; \mathbf{P}_{0}\right)$. Hence there exists a $L^{\infty}\left(I ; \mathbf{P}^{0}\right)$-weakly*-subsequential limit $\tilde{\mathbf{p}} \in C\left(\bar{I} ; \mathbf{P}^{0}\right)$. Utilizing the governing equation for $\mathbf{p}_{\varepsilon}$ we find that $\left(\mathbf{p}_{\varepsilon, 1}\right)_{t}=-\tau_{\varepsilon} \Delta \mathbf{p}_{\varepsilon, 2},\left(\mathbf{p}_{\varepsilon, 2}\right)_{t}=-\tau_{\varepsilon} \mathbf{p}_{\varepsilon, 1}$, and hence we can apply an Arzela-Ascoli argument to $\left\{\mathbf{p}_{\varepsilon}\right\}$ considered as family in $C\left(\bar{I} ; \mathbf{P}^{-1}\right)$ to argue that subsequentially $\mathbf{p}_{\varepsilon} \rightarrow \tilde{\mathbf{p}}$ in $C\left(\bar{I} ; \mathbf{P}^{-1}\right)$. In particular this implies that $\mathbf{p}_{\varepsilon, 2}(1) \rightarrow \tilde{\mathbf{p}}_{2}(1)$ in $H^{-1}(\Omega)$, and, possibly on a further subsequence

$$
\mathbf{p}_{\varepsilon, 2}(1) \rightarrow \tilde{\mathbf{p}}_{2}(1) \text { in } L^{2}(\Omega) .
$$

Let us show that the weak limit $\tilde{\mathbf{p}}$ satisfies (4.4) and (4.5). Passing to the limit in the first and second equations of (3.4) and using (4.3) and Corollary 3.3 we obtain the first two equations of (4.4). Since under the bang-bang assumption we have strong subsequential convergence of $\left\{u_{\varepsilon}\right\}$ to $\tilde{u}$ in $L^{2}(\omega)$ we can pass to the limit in the maximum principle inequality of (3.4) to obtain the maximum principle in (4.4). 
We next argue that $\tilde{\mathbf{p}}(1)$ or, equivalently, that $\tilde{\mathbf{p}}$ is nontrivial. Due to controllability with $T<\tau^{*}$ there exists $\hat{\varepsilon} \in(0, \bar{\varepsilon}]$ such that for $\varepsilon \in(0, \hat{\varepsilon}]$ the system is controllable for all $\tau_{\varepsilon}$, and there exists a constant $\bar{c}$ such that

$$
\left\|\mathbf{p}_{\varepsilon}(1)\right\|_{\mathbf{P}^{0}}^{2} \leq \bar{c}\left\|\mathbf{B}^{*} \mathbf{p}_{\varepsilon}\right\|_{L^{2}\left(I ; L^{2}(\omega)\right)}^{2} \leq \bar{c}\left\|\mathbf{B}^{*} \mathbf{p}_{\varepsilon}\right\|_{L^{1}\left(I ; L^{2}(\omega)\right)}\left\|\mathbf{B}^{*} \mathbf{p}_{\varepsilon}\right\|_{L^{\infty}\left(I ; L^{2}(\omega)\right)} .
$$

Combining this estimate with Lemma 4.2 we obtain for $\varepsilon \in(0, \hat{\varepsilon}]$

$$
\delta^{2} \leq \bar{c}\left\|\mathbf{B}^{*} \mathbf{p}_{\varepsilon}\right\|_{L^{1}\left(I ; L^{2}(\omega)\right)}\left\|\mathbf{B}^{*} \mathbf{p}_{\varepsilon}\right\|_{L^{\infty}\left(I ; L^{2}(\omega)\right)}
$$

By Lemma 4.1 we obtain

$$
\begin{aligned}
\gamma\left\|\mathbf{B}^{*} \mathbf{p}_{\varepsilon}\right\|_{L^{1}\left(I ; L^{2}(\omega)\right)} & =\gamma\left\|-\varepsilon u_{\varepsilon}+\varepsilon u_{\varepsilon}+\mathbf{B}^{*} \mathbf{p}_{\varepsilon}\right\|_{L^{1}\left(I ; L^{2}(\omega)\right)} \\
& \leq \gamma \varepsilon\left\|u_{\varepsilon}\right\|_{L^{1}\left(I ; L^{2}(\omega)\right)}+\gamma\left\|\varepsilon u_{\varepsilon}+\mathbf{B}^{*} \mathbf{p}_{\varepsilon}\right\|_{L^{1}\left(I ; L^{2}(\omega)\right)} \\
& =\gamma \varepsilon\left\|u_{\varepsilon}\right\|_{L^{1}\left(I ; L^{2}(\omega)\right)}-\varepsilon\left\|u_{\varepsilon}\right\|_{L^{2}\left(I ; L^{2}(\omega)\right)}^{2}-\left(u_{\varepsilon}, \mathbf{B}^{*} \mathbf{p}_{\varepsilon}\right)_{L^{2}\left(I ; L^{2}(\omega)\right)}
\end{aligned}
$$

Combined with (4.9) this implies that for $\varepsilon \in(0, \hat{\varepsilon}]$

$$
\delta^{2} \leq \frac{\bar{c}}{\gamma}\left\|\mathbf{B}^{*} \mathbf{p}_{\varepsilon}\right\|_{L^{\infty}\left(I ; L^{2}(\omega)\right)}\left(\gamma \varepsilon \sigma_{\varepsilon}\left\|u_{\varepsilon}\right\|_{L^{1}\left(I ; L^{2}(\omega)\right)}-\varepsilon \sigma_{\varepsilon}\left\|u_{\varepsilon}\right\|_{L^{2}\left(I ; L^{2}(\omega)\right)}-\left(u_{\varepsilon}, \mathbf{B}^{*} \mathbf{p}_{\varepsilon}\right)_{L^{2}\left(I ; L^{2}(\omega)\right)}\right) .
$$

The first and the second addend in the brackets on the right hand side tend to zero for $\varepsilon \rightarrow 0^{+}$, and the third addend is convergent. If the weak subsequential limit of $\mathbf{p}_{\varepsilon}$ in $L^{2}\left(I ; L^{2}(\omega)\right)$ was zero this contradicts the above inequality and hence $\tilde{\mathbf{p}}$ is nontrivial.

We next argue that (4.5) holds. Since by assumption $\mathbf{B}^{*} \tilde{\mathbf{p}}(1)$ is nontrivial and since $\tilde{\mathbf{p}} \in C\left(\bar{I} ; \mathbf{P}^{0}\right)$ there exists a left-sided neighborhood $\tilde{U}$ of 1 such that $\mathbf{B}^{*} \tilde{\mathbf{p}}(t) \neq 0$ for $t \in \tilde{U}$. Hence

$$
\tilde{u}(t)=-\gamma \frac{\mathbf{B}^{*} \tilde{\mathbf{p}}(t)}{\left\|\mathbf{B}^{*} \tilde{\mathbf{p}}(t)\right\|_{L^{2}(\omega)}} \text { for } t \in \tilde{U}
$$

and in particular $\tilde{u}$ is continuous from $\tilde{U}$ to $\mathbf{P}^{0}$ and hence $\tilde{u}(1)$ is well-defined. We have

$$
\gamma=\|\tilde{u}(1)\|_{L^{2}(\omega)} \geq \limsup \left\|\tilde{u}_{\varepsilon}(1)\right\|_{L^{2}(\omega)} \geq \liminf \left\|\tilde{u}_{\varepsilon}(1)\right\|_{L^{2}(\omega)} \geq\|\tilde{u}(1)\|_{L^{2}(\omega)}=\gamma,
$$

and hence

$$
u_{\varepsilon}(1) \rightarrow \tilde{u}(1) \text { in } L^{2}(\omega) .
$$

We can now pass to the limit $\varepsilon \rightarrow 0^{+}$in (3.7) to obtain

$$
1+\langle\mathbf{A} z+\mathbf{B} \tilde{u}(1), \mathbf{p}(1)\rangle_{\left(\mathbf{P}^{0}\right)^{*}, \mathbf{P}^{0}}=0,
$$

where we use (4.7) and (4.10). This ends the proof of part (a).

We now turn to the case that $\left\{\mathbf{p}_{\varepsilon}(1)\right\}$ is unbounded in $\mathbf{P}^{0}$. In this case we consider

$$
\tilde{\mathbf{p}}_{\varepsilon}=\frac{\mathbf{p}_{\varepsilon}(1)}{\left\|\mathbf{p}_{\varepsilon}(1)\right\|_{\mathbf{P}^{0}}} .
$$

This sequence contains a weakly convergent subsequence in $\mathbf{P}^{0}$ with limit $\tilde{\mathbf{p}}(1) \in \mathbf{P}^{0}$. Let $\tilde{\mathbf{p}}_{\varepsilon}$ and $\tilde{\mathbf{p}} \in C\left(I ; \mathbf{P}^{0}\right)$ denote the solutions of the adjoint equations with terminal conditions $\tilde{\mathbf{p}}_{\varepsilon}(1)$ and $\tilde{\mathbf{p}}(1)$, respectively. We can now proceed in exactly the same manner as above for case (a) to verify (4.4). To verify non-triviality of $\tilde{\mathbf{p}}$ which in this case is the $L^{\infty}\left(I ; \mathbf{P}^{0}\right)$-weakly*-subsequential limit of $\tilde{\mathbf{p}}_{\varepsilon}$ we modify (4.8) which leads to

$$
1=\left\|\tilde{\mathbf{p}}_{\varepsilon}(1)\right\|_{\mathbf{P}^{0}}^{2} \leq \bar{c}\left\|\mathbf{B}^{*} \tilde{\mathbf{p}}_{\varepsilon}\right\|_{L^{2}\left(I ; L^{2}(\omega)\right)}^{2} \leq \bar{c}\left\|\mathbf{B}^{*} \tilde{\mathbf{p}}_{\varepsilon}\right\|_{L^{1}\left(I ; L^{2}(\omega)\right)}\left\|\mathbf{B}^{*} \tilde{\mathbf{p}}_{\varepsilon}\right\|_{L^{\infty}\left(I ; L^{2}(\omega)\right)}
$$


for $\varepsilon \in(0, \hat{\varepsilon}]$. By Lemma 4.1 we have

$$
\left(u_{\varepsilon}, \mathbf{B}^{*} \tilde{\mathbf{p}}_{\varepsilon}\right)_{L^{2}\left(I ; L^{2}(\omega)\right)}=-\varepsilon \sigma_{\varepsilon}\left\|u_{\varepsilon}\right\|_{L^{2}\left(I ; L^{2}(\omega)\right)}^{2}-\gamma\left\|\varepsilon \sigma_{\varepsilon} u_{\varepsilon}+\mathbf{B}^{*} \tilde{\mathbf{p}}_{\varepsilon}\right\|_{L^{1}\left(I ; L^{2}(\omega)\right)},
$$

where $\sigma_{\varepsilon}=\frac{1}{\left\|\mathbf{p}_{\varepsilon}(1)\right\|_{\mathbf{P}^{0}}}$, which is is bounded due to Lemma 4.2. This implies that

$$
\begin{aligned}
\gamma\left\|\mathbf{B}^{*} \tilde{\mathbf{p}}_{\varepsilon}\right\|_{L^{1}\left(I ; L^{2}(\omega)\right)} & =\gamma\left\|-\varepsilon \sigma_{\varepsilon} u_{\varepsilon}+\varepsilon \sigma_{\varepsilon} u_{\varepsilon}+\mathbf{B}^{*} \tilde{\mathbf{p}}_{\varepsilon}\right\|_{L^{1}\left(I ; L^{2}(\omega)\right)} \\
& \leq \gamma \varepsilon \sigma_{\varepsilon}\left\|u_{\varepsilon}\right\|_{L^{1}\left(I ; L^{2}(\omega)\right)}+\gamma\left\|\varepsilon \sigma_{\varepsilon} u_{\varepsilon}+\mathbf{B}^{*} \tilde{\mathbf{p}}_{\varepsilon}\right\|_{L^{1}\left(I ; L^{2}(\omega)\right)} \\
& =\gamma \varepsilon \sigma_{\varepsilon}\left\|u_{\varepsilon}\right\|_{L^{1}\left(I ; L^{2}(\omega)\right)}-\varepsilon \sigma_{\varepsilon}\left\|u_{\varepsilon}\right\|_{L^{2}\left(I ; L^{2}(\omega)\right)}^{2}-\left(u_{\varepsilon}, \mathbf{B}^{*} \tilde{\mathbf{p}}_{\varepsilon}\right)_{L^{2}\left(I ; L^{2}(\omega)\right)} .
\end{aligned}
$$

Combining the last two estimates we find for $\varepsilon \in(0, \hat{\varepsilon}]$

$$
1 \leq \frac{\bar{c}}{\gamma}\left\|\mathbf{B}^{*} \tilde{\mathbf{p}}_{\varepsilon}\right\|_{L^{\infty}\left(I ; L^{2}(\omega)\right)}\left(\gamma \varepsilon \sigma_{\varepsilon}\left\|u_{\varepsilon}\right\|_{L^{1}\left(I ; L^{2}(\omega)\right)}-\varepsilon \sigma_{\varepsilon}\left\|u_{\varepsilon}\right\|_{L^{2}\left(I ; L^{2}(\omega)\right)}-\left(u_{\varepsilon}, \mathbf{B}^{*} \tilde{\mathbf{p}}_{\varepsilon}\right)_{L^{2}\left(I ; L^{2}(\omega)\right)}\right) .
$$

The first and the second addend in the brackets on the right hand side tend to zero for $\varepsilon \rightarrow 0^{+}$, and the third addend is convergent. If the weak subsequential limit of $\mathbf{B}^{*} \tilde{\mathbf{p}}_{\varepsilon}$ in $L^{2}\left(I ; L^{2}(\omega)\right)$ was zero this would contradict the above inequality and hence $\mathbf{B}^{*} \tilde{\mathbf{p}}$ and in particular $\tilde{\mathbf{p}}$ are non-trivial.

From (3.7) after taking the quotient with $\left\|\mathbf{p}_{\varepsilon}(1)\right\|_{\mathbf{P}^{0}}$, which tends to infinity for $\varepsilon \rightarrow 0$, we obtain

$$
\langle\mathbf{A z}+\mathbf{B} \tilde{u}(1), \tilde{\mathbf{p}}(1)\rangle_{\left(\mathbf{P}^{0}\right)^{*}, \mathbf{P}^{0}}=0 .
$$

If $\mathbf{z}=0$ then $\langle\mathbf{B} \tilde{u}(1), \tilde{\mathbf{p}}(1)\rangle_{\left(\mathbf{P}^{0}\right)^{*}, \mathbf{P}^{0}}=-\gamma\left\|\tilde{\mathbf{p}}_{2}(1)\right\|_{L^{2}(\omega)}=0$, which gives a contradiction.

Theorem 4.3 provides sufficient conditions guaranteeing that the optimality system (4.4) is qualified, i.e. $\tilde{\mathbf{p}} \neq 0$. Moreover

$$
\tilde{u}(t)=-\gamma \frac{\mathbf{B}^{*} \tilde{\mathbf{p}}(t)}{\left\|\mathbf{B}^{*} \tilde{\mathbf{p}}(t)\right\|_{L^{2}(\omega)}} \text { for } \mathbf{B}^{*} \tilde{\mathbf{p}}(t) \neq 0 .
$$

The requirement that system (2.1) is controllable for some $T<\tau^{*}$ is a plausible one: since controllability refers to controls without control constraints whereas the optimal time $\tau^{*}$ must be achieved with constrained controls, in most cases it can be expected that the smallest time for which unconstrained controllability holds is typically strictly smaller than $\tau^{*}$.

Theorem 4.4. Suppose that (2.1) is controllable for some $T<\tau^{*}$ and that $\left\{\mathbf{p}_{\varepsilon}(1)\right\}$ is bounded in $\mathbf{P}^{1}$. Then there exists a non-trivial $\tilde{\mathbf{p}} \in C\left(I ; \mathbf{P}^{1}\right)$ such that

$$
\left\{\begin{array}{l}
\tilde{\mathbf{y}}_{t}=\tau^{*} \mathbf{A} \tilde{\mathbf{y}}+\tau^{*} \mathbf{B} \tilde{u}, \tilde{\mathbf{y}}(0)=\mathbf{y}_{0}, \tilde{\mathbf{y}}(1)=\mathbf{z}, \\
-\tilde{\mathbf{p}}_{t}=\tau^{*} \mathbf{A}^{*} \tilde{\mathbf{p}}, \\
\left(\mathbf{B}^{*} \tilde{\mathbf{p}}, u-\tilde{u}\right)_{L^{2}\left(I ; L^{2}(\omega)\right)} \geq 0 \text { for all } u \in U_{a d}, \\
1+\langle\mathbf{A z}+\mathbf{B} \tilde{u}(1), \tilde{\mathbf{p}}(1)\rangle_{\left(\mathbf{P}^{0}\right)^{*}, \mathbf{P}^{0}}=0 .
\end{array}\right.
$$

Proof. The equations in the first two lines of (4.11) can be verified as in the proof of Theorem 4.3.

To prove the maximum principle in $(4.11)$, we have to show the convergence $\left(\mathbf{B}^{*} \mathbf{p}_{\varepsilon}, u_{\varepsilon}\right)_{L^{2}\left(I ; L^{2}(\omega)\right)} \rightarrow$ $\left(\mathbf{B}^{*} \tilde{\mathbf{p}}, \tilde{u}\right)_{L^{2}\left(I ; L^{2}(\omega)\right)}$. Since $\left\{\mathbf{p}_{\varepsilon}(1)\right\}_{\varepsilon}$ is assumed to be bounded in $\mathbf{P}^{1}$ we have weak convergence of another subsequence $\mathbf{p}_{\varepsilon}(1) \rightarrow \tilde{\mathbf{p}}(1)$ in $\mathbf{P}^{1}$, which implies weak* convergence of $\mathbf{p}_{\varepsilon} \rightarrow^{*} \tilde{\mathbf{p}}$ in $L^{\infty}\left(I ; \mathbf{P}^{1}\right) \cap H^{1}\left(I ; \mathbf{P}^{0}\right)$ to $\tilde{\mathbf{p}} \in C\left(I ; \mathbf{P}^{1}\right) \cap H^{1}\left(I ; \mathbf{P}^{0}\right)$. Arzela-Ascoli's theorem and the compact embedding $\mathbf{P}^{1}$ in $\mathbf{P}^{0}$ imply that $\mathbf{p}_{\varepsilon} \rightarrow \tilde{\mathbf{p}}$ in $C\left(I ; \mathbf{P}^{0}\right)$ as $\varepsilon \rightarrow 0$. Hence for a subsequence

$$
\left(\mathbf{B}^{*} \mathbf{p}_{\varepsilon}, u_{\varepsilon}\right)_{L^{2}\left(I ; L^{2}(\omega)\right)} \rightarrow\left(\mathbf{B}^{*} \tilde{\mathbf{p}}, \tilde{u}\right)_{L^{2}\left(I ; L^{2}(\omega)\right)}
$$

This allows us to pass to the limit in the third line of (3.4) to obtain the third line of (4.11). 
Non-triviality of $\tilde{\mathbf{p}}$ is argued exactly as in the proof of Theorem 4.3 part (a).

Finally we pass to the limit in (3.7). If on the one hand $\mathbf{B}^{*} \tilde{\mathbf{p}}(1)=0$, then we have $\left(u_{\varepsilon}(1), \mathbf{B}^{*} \mathbf{p}_{\varepsilon}(1)\right) \rightarrow 0=$ $\left(\tilde{u}(1), \mathbf{B}^{*} \tilde{\mathbf{p}}(1)\right)$. If on the other hand $\mathbf{B}^{*} \tilde{\mathbf{p}}(1) \neq 0$, then there exist $\tilde{\varepsilon}, \delta, \alpha$ such that $\left\|\mathbf{B}^{*} \mathbf{p}_{\varepsilon}(t)\right\|_{L^{2}(\omega)} \geq \alpha>0$, for all $\varepsilon \in(0, \tilde{\varepsilon}), t \in[1-\delta, 1]$. Then $u_{\varepsilon}(t)=\gamma \frac{\mathbf{B}^{*} \mathbf{p}_{\varepsilon}(t)}{\left\|\mathbf{B}^{*} \mathbf{p}_{\varepsilon}(t)\right\|_{L^{2}(\omega)}} \rightarrow \gamma \frac{\mathbf{B}^{*} \tilde{\mathbf{p}}(t)}{\left\|\mathbf{B}^{*} \tilde{\mathbf{p}}(t)\right\|_{L^{2}(\omega)}}$ strongly in $L^{2}(\omega)$ as $\varepsilon \rightarrow 0^{+}$for all $t \in[1-\delta, 1]$. Hence $\left(u_{\varepsilon}(1), \mathbf{B}^{*} \mathbf{p}_{\varepsilon}(1)\right)_{L^{2}(\omega)} \rightarrow\left(\tilde{u}(1), \mathbf{B}^{*} \tilde{\mathbf{p}}(1)\right)_{L^{2}(\omega)}$ holds. We can now pass to the limit in (3.7) to obtain the transversality condition.

Remark 4.5. If $\left|\tau_{\varepsilon}-\tau^{*}\right|=O(\varepsilon)$, which can be observed in certain numerical experiments, see Section 6 below, then due to Corollary 3.3 and the relation $\left\|\mathbf{p}_{\varepsilon}(1)\right\|_{\mathbf{P}^{1}}=\frac{1}{\varepsilon}\left\|\mathbf{y}_{\varepsilon}(1)-\mathbf{z}\right\|_{\mathbf{Y}^{0}}$ the sequence $\left\{\mathbf{p}_{\varepsilon}(1)\right\}$ is bounded in $\mathbf{P}^{1}$.

In order to pass to the limit in the integrated form of the transversality condition we need additionally to assume that $\tilde{u}$ is bang-bang.

Corollary 4.6. If in addition to the assumptions of the previous theorem $\tilde{u}$ is bang-bang then

$$
1+\langle\mathbf{A} \tilde{\mathbf{y}}+\mathbf{B} \tilde{u}, \tilde{\mathbf{p}}\rangle_{L^{2}\left(I ;\left(\mathbf{P}^{1}\right)^{*}\right), L^{2}\left(I ; \mathbf{P}^{1}\right)}=0 .
$$

Proof. If $\tilde{u}$ is bang-bang, then $u_{\varepsilon} \rightarrow \tilde{u}$ strongly in $L^{2}\left(I ; L^{2}(\omega)\right)$ by Theorem 3.2 in addition to (4.3). Moreover, we have $\mathbf{y}_{\varepsilon} \rightarrow \tilde{\mathbf{y}}$ strongly in $C\left(I ; \mathbf{Y}^{1}\right)$. Hence, it holds $\mathbf{A y}_{\varepsilon}+\mathbf{B} u_{\varepsilon} \rightarrow \mathbf{A} \tilde{\mathbf{y}}+\mathbf{B} \tilde{u}$ strongly in $L^{2}\left(I ; \mathbf{Y}^{0}\right)=L^{2}\left(I ;\left(\mathbf{P}^{1}\right)^{*}\right)$. If $\left\{\mathbf{p}_{\varepsilon}(1)\right\}_{\varepsilon \in(0,1]}$ is bounded in $\mathbf{P}^{1}$, then we have $\mathbf{p}_{\varepsilon} \rightarrow \tilde{\mathbf{p}}$ in $L^{2}\left(I ; \mathbf{P}^{1}\right)$. Hence, we can pass to the limit in the last equation of (3.4) to obtain the claim.

Finally we have constancy of the Hamiltonian along optimal trajectories.

Corollary 4.7 (pointwise transversality). If in addition to the assumptions of the previous theorem $\tilde{u}$ is bangbang and $\mathbf{y}_{0} \in \mathbf{Y}^{2}$ then

$$
\mathcal{H}(\tilde{\mathbf{y}}(t), \tilde{u}(t), \tilde{\mathbf{p}}(t))=0 \text { for almost all } t \in \bar{I} .
$$

Proof. From Corollary 3.8 we recall that

$$
1+\frac{\varepsilon}{2}\left\|u_{\varepsilon}(t)\right\|_{L^{2}(\omega)}^{2}+\left\langle\mathbf{A} \mathbf{y}_{\varepsilon}(t)+\mathbf{B} u_{\varepsilon}(t), \mathbf{p}_{\varepsilon}(t)\right\rangle_{\mathbf{Y}^{0}, \mathbf{P}^{1}}=0
$$

for all $t \in(0,1)$. As in the proof of Corollary 4.6 we use that $\tilde{u}$ is assumed to be bang-bang. Then $u_{\varepsilon} \rightarrow \tilde{u}$ strongly in $L^{2}\left(I ; L^{2}(\omega)\right)$, and hence $\mathbf{y}_{\varepsilon} \rightarrow \tilde{\mathbf{y}}$ strongly in $C\left(I ; \mathbf{Y}^{1}\right)$ and $\mathbf{A y}_{\varepsilon} \rightarrow \mathbf{A} \tilde{\mathbf{y}}$ strongly in $C\left(I ; \mathbf{Y}^{0}\right)=C\left(I ;\left(\mathbf{P}^{1}\right)^{*}\right)$. From the proof of Theorem 4.4 we have a subsequence for which $\mathbf{p}_{\varepsilon} \rightarrow \tilde{\mathbf{p}}$ in $C\left(I ; \mathbf{P}^{0}\right)$. Since $\left\{\mathbf{p}_{\varepsilon}(t)\right\}$ is bounded in $\mathbf{P}^{1}$ for $\varepsilon \rightarrow 0$ for every $t \in I$ we have $\mathbf{p}_{\varepsilon}(t) \rightarrow \tilde{\mathbf{p}}(t)$ in $\mathbf{P}^{1}$ for $\varepsilon \rightarrow 0$ for each $t \in I$. Hence, we can pass to the limit in (4.13) for almost every $t \in I$ to obtain the claim.

Remark 4.8. Our formulation of the regularized problems $\left(P_{\varepsilon}\right)$ realizes the terminal constraint by means of penalty. We could alternatively consider

$$
\left\{\begin{array}{l}
\min \tau\left(1+\frac{\varepsilon}{2}\|u\|_{L^{2}\left(I ; L^{2}(\omega)\right)}^{2}\right) \\
\text { subject to } \tau \geq 0, \text { and } \\
\mathbf{y}_{t}=\tau \mathbf{A} \mathbf{y}+\tau \mathbf{B} u, \text { on }(0,1] \\
\mathbf{y}(0)=\mathbf{y}_{0}, \mathbf{y}(1)=\mathbf{z}, u \in U_{a d}
\end{array}\right.
$$

where the terminal condition is kept as explicit constraint. For every $\varepsilon>0$ problem (4.14) has a solution $\left(\tau_{\varepsilon}, y_{\varepsilon}, u_{\varepsilon}\right)$ which satisfies the monotonicity properties, for $0<\varepsilon_{0}<\varepsilon_{1}$,

$$
\tau^{*} \leq \tau_{\varepsilon_{0}} \leq \tau_{\varepsilon_{1}} \leq \tau^{*}\left(1+\frac{\gamma \varepsilon_{1}}{2}\right)
$$


and

$$
\left\|u_{\varepsilon_{1}}\right\|_{L^{2}\left(I ; L^{2}(\omega)\right)} \leq\left\|u_{\varepsilon_{0}}\right\|_{L^{2}\left(I ; L^{2}(\omega)\right)} \leq\left\|u^{*}\right\|_{L^{2}\left(I ; L^{2}(\omega)\right)},
$$

which can be verified with arguments analogous to those in [11]. Developing a Lagrangian theory for (4.14), however, is impeded by the fact that the constraints in (4.14) are not differentiable in the natural norms. Specifically we define

$$
e(\tau, u): \mathbb{R} \times L^{2}\left(I ; L^{2}(\omega)\right) \rightarrow \mathbf{Y}^{1}
$$

given by $e(\tau, u)=\mathbf{y}(\tau, u)(1)=\left(y(\tau, u)(1), y_{t}(\tau, u)(1)\right)$. Note that $e$ is not differentiable with respect to $\tau$. In fact, $e_{\tau}(\tau, u)$ would be the solution to

$$
\begin{aligned}
& \tilde{\mathbf{y}}_{t}=\tau \mathbf{A} \tilde{\mathbf{y}}+\mathbf{A y}+\mathbf{B} u \\
& \tilde{\mathbf{y}}(0)=0
\end{aligned}
$$

evaluated at 1 . Since $\mathbf{A y} \in C\left(I ; \mathbf{Y}^{0}\right)$ only, this does not guarantee that $\tilde{\mathbf{y}}(1) \in \mathbf{Y}^{1}$, in general.

\section{Adapted penalty method}

In the previous section, optimality conditions are presented that are satisfied by accumulation points of solutions $\left(\tau_{\varepsilon}, \mathbf{y}_{\varepsilon}, u_{\varepsilon}\right)$ to $\left(P_{\varepsilon}\right)$. This does not yet guarantee that every solution $\left(\tau^{*}, \mathbf{y}^{*}, u^{*}\right)$ to $(\tilde{P})$ satisfies such a condition. Here, we present a partial result addressing this question. To this end, we utilize an adapted penalty technique.

For a fixed solution $\left(\tau^{*}, \mathbf{y}^{*}, u^{*}\right)$ to $(\tilde{P})$ we consider

$$
\left\{\begin{array}{l}
\min \hat{J}_{\varepsilon}(\tau, u)=\tau\left(1+\frac{\varepsilon}{2}\|u\|_{L^{2}\left(I ; L^{2}(\omega)\right)}^{2}\right)+\frac{1}{2 \varepsilon}\|\mathbf{y}(1)-\mathbf{z}\|_{\mathbf{Y}^{0}}^{2}+\frac{\tau}{2}\left\|u-u^{*}\right\|_{L^{2}\left(I ; L^{2}(\omega)\right)}^{2}, \\
\text { subject to } \tau \geq 0 \text { and } \\
\mathbf{y}_{t}=\tau \mathbf{A y}+\tau \mathbf{B} u, \text { on }(0,1], \\
\mathbf{y}(0)=\mathbf{y}_{0}, \\
u \in U_{a d} .
\end{array}\right.
$$

Note that the adapted penalty formulation $\left(\hat{P}_{\varepsilon}\right)$, differently from $\left(P_{\varepsilon}\right)$, uses the solution $u^{*}$. Thus it is not suited for numerical realization of the time optimal control problem.

Proposition 5.1. For every $\varepsilon>0$ there exists a solution $\left(\tau_{\varepsilon}, \mathbf{y}_{\varepsilon}, u_{\varepsilon}\right)$ of $\left(\hat{P}_{\varepsilon}\right)$ and $\left(\tau_{\varepsilon}, \mathbf{y}_{\varepsilon}, u_{\varepsilon}\right) \rightarrow\left(\tau^{*}, \mathbf{y}^{*}, u^{*}\right)$ as $\varepsilon \rightarrow 0^{+}$strongly in $\mathbb{R} \times\left(C\left(\bar{I} ; \mathbf{Y}^{1}\right) \cap H^{1}\left(I ; \mathbf{Y}^{0}\right)\right) \times L^{2}\left(I ; L^{2}(\omega)\right)$. Moreover there exists $\mathbf{p}_{\varepsilon} \in C\left(\bar{I} ; \mathbf{P}^{2}\right) \cap C^{1}\left(I ; \mathbf{P}^{1}\right)$ such that the following optimality system holds:

$$
\left\{\begin{array}{l}
\partial_{t} \mathbf{y}_{\varepsilon}=\tau_{\varepsilon} \mathbf{A y}_{\varepsilon}+\tau_{\varepsilon} \mathbf{B} u_{\varepsilon}, \quad \mathbf{y}_{\varepsilon}(0)=\mathbf{y}_{0}, \\
-\partial_{t} \mathbf{p}_{\varepsilon}=\tau_{\varepsilon} \mathbf{A}^{*} \mathbf{p}_{\varepsilon}, \quad \mathbf{p}_{\varepsilon}(1)=\frac{1}{\varepsilon}\left(\begin{array}{c}
\mathbf{y}_{\varepsilon, 1}(1)-z_{1} \\
(-\Delta)^{-1}\left(\mathbf{y}_{\varepsilon, 2}(1)-z_{2}\right)
\end{array}\right) \in \mathbf{P}^{2}, \\
\left(\varepsilon u_{\varepsilon}+\mathbf{B}^{*} \mathbf{p}_{\varepsilon}+u_{\varepsilon}-u^{*}, u-u_{\varepsilon}\right)_{L^{2}\left(I ; L^{2}(\omega)\right)} \geq 0, \text { for all } u \in U_{a d}, \\
1+\frac{\varepsilon}{2}\left\|u_{\varepsilon}\right\|_{L^{2}\left(I ; L^{2}(\omega)\right)}^{2}+\frac{1}{2}\left\|u_{\varepsilon}-u^{*}\right\|_{L^{2}\left(I ; L^{2}(\omega)\right)}^{2}+\left\langle\mathbf{A} \mathbf{y}_{\varepsilon}+\mathbf{B} u_{\varepsilon}, \mathbf{p}_{\varepsilon}\right\rangle_{L^{2}\left(I ; \mathbf{Y}^{0}\right), L^{2}\left(I ; \mathbf{P}^{1}\right)}=0 .
\end{array}\right.
$$

Proof. For the most part we can follow the proofs of Theorems 3.2 and 3.6 and we only provide the necessary changes. Since $\left(\tau^{*}, \mathbf{y}^{*}, u^{*}\right)$ is feasible for $\left(\hat{P}_{\varepsilon}\right)$ we have

$$
\tau_{\varepsilon}\left(1+\frac{\varepsilon}{2}\left\|u_{\varepsilon}\right\|_{L^{2}\left(I ; L^{2}(\omega)\right)}^{2}\right)+\frac{1}{2 \varepsilon}\left\|\mathbf{y}_{\varepsilon}(1)-\mathbf{z}\right\|_{\mathbf{Y}^{0}}^{2}+\frac{\tau_{\varepsilon}}{2}\left\|u_{\varepsilon}-u^{*}\right\|_{L^{2}\left(I ; L^{2}(\omega)\right)}^{2} \leq \tau^{*}\left(1+\frac{\varepsilon}{2}\left\|u^{*}\right\|_{L^{2}\left(I ; L^{2}(\omega)\right)}^{2}\right) .
$$

This implies that $\left(\tau_{\varepsilon}, \mathbf{y}_{\varepsilon}, u_{\varepsilon}\right) \rightarrow\left(\tau^{*}, \mathbf{y}^{*}, u^{*}\right)$ in $\mathbb{R} \times\left(C\left(\bar{I} ; \mathbf{Y}^{1}\right) \cap H^{1}\left(I ; \mathbf{Y}^{0}\right)\right) \times L^{2}\left(I ; L^{2}(\omega)\right)$. Arguing as in the proof of Theorem 3.6 we find (5.1). 
Let us note that a result similar to Corollary 3.8 cannot be expected, since the optimal control problem $\left(\hat{P}_{\varepsilon}\right)$ is non-autonomous. This is due to the fact that the cost function $\hat{J}_{\varepsilon}(\tau, u)$ can be written as

$$
\hat{J}_{\varepsilon}(\tau, u)=\int_{0}^{1} f_{0}(t, u(t), \tau) \mathrm{d} t+g_{0}(\mathbf{y}(1)),
$$

where the integrand $f_{0}$ depends on $t \in I$ via the penalty term that involves $u^{*}$. In order to derive a result similar to Corollary 3.11, one could aim at adapting techniques for non-autonomous optimal control problems for ordinary differential equations, which is beyond the scope of this paper.

Let us now present the resulting necessary optimality condition under conditions similar to those of Theorem 4.4 .

Theorem 5.2. Let $\left(\tau^{*}, \mathbf{y}^{*}, u^{*}\right)$ denote a solution to $(\tilde{P})$. Suppose that (2.1) is controllable for some $T<\tau^{*}$ and that $\left\{\mathbf{p}_{\varepsilon}(1)\right\}$ is bounded in $\mathbf{P}^{1}$. Then there exists a non-trivial $\mathbf{p}^{*} \in C\left(I ; \mathbf{P}^{1}\right)$ such that

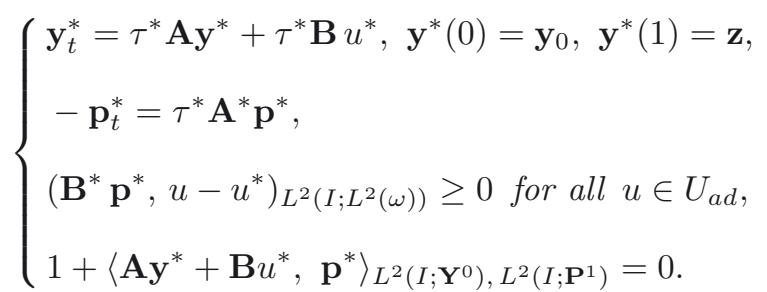

Proof. Since $\left\{\mathbf{p}_{\varepsilon}(1)\right\}_{\varepsilon}$ is assumed to be bounded in $\mathbf{P}^{1}$, we have weak convergence of a subsequence $\mathbf{p}_{\varepsilon}(1) \rightarrow$ $\mathbf{p}^{*}(1)$ in $\mathbf{P}^{1}$, which implies weak* convergence of $\mathbf{p}_{\varepsilon} \rightarrow^{*} \mathbf{p}^{*}$ in $L^{\infty}\left(I ; \mathbf{P}^{1}\right) \cap H^{1}\left(I ; \mathbf{P}^{0}\right)$ to $\mathbf{p}^{*} \in C\left(I ; \mathbf{P}^{1}\right) \cap$ $H^{1}\left(I ; \mathbf{P}^{0}\right)$. Using convergence of $\left(\tau_{\varepsilon}, \mathbf{y}_{\varepsilon}, u_{\varepsilon}\right) \rightarrow\left(\tau^{*}, \mathbf{y}^{*}, u^{*}\right)$ as proven in Proposition 5.1 , we can pass to the limit $\varepsilon \rightarrow 0^{+}$in (5.1) to obtain (5.3). From the last equation of (5.3) it follows that $\mathbf{p}^{*} \neq 0$.

\section{NumericAl EXPERIMENT}

We report on a numerical experiment to solve the time-optimal control problem by means of the regularized problem $\left(P_{\varepsilon}\right)$. It shows that $\left\{\mathbf{p}_{\varepsilon}(1)\right\}$ is bounded in $\mathbf{P}^{1}$, i.e. the the essential requirement of Theorem 4.4 to obtain the transversality condition in (4.11) in the limit $\varepsilon \rightarrow 0$ can be fulfilled. A more detailed report on the numerical approach and further examples will be given elsewhere.

We chose $\Omega=(0,1)^{2}$, and the control bound was set to $\gamma=3$. The target state was $z_{1}=z_{2}=0$ and the initial state was given as $y_{1}\left(x_{1}, x_{2}\right)=x_{1} x_{2}\left(1-x_{1}\right)\left(1-x_{2}\right)$, the initial velocity was set to $y_{2}=0$. The control domain was chosen to be $\omega:=\Omega \backslash[0,0.1]^{2}$. This implies that the geometric control condition of [4] is satisfied. In particular, we have null-controllability of the wave equation for all $\tau>\frac{\sqrt{2}}{10} \approx 0.1414$. Moreover, due to Theorem 2.4, see also [18], Theorem 3.1, the time-optimal control problem is solvable for these data.

The spatial domain was discretized using a uniform triangulation, and the time interval was split into equidistant subintervals. We will report on the results for the following hierarchy of discretizations: $(N, M)=(50,10)$, $(200,20),(800,40)$, and $(3200,80)$, where $N$ is the numbers of triangles and $M$ the numbers of time intervals. The resulting mesh size $h$ is $h=2 / \sqrt{N}$, the resulting length of the temporal subintervals $\Delta t=1 / M$.

We utilized a path-following strategy to drive $\varepsilon$ from the initial value $\varepsilon_{0}=0.1$ towards zero. This iteration was stopped as soon as the terminal residual satisfied $\left\|\mathbf{y}_{\varepsilon}(1)-\mathbf{z}\right\|_{\mathbf{Y}^{0}} \leq 10^{-3}$. The algorithmic details will be given independently.

We report on the convergence for $\varepsilon \rightarrow 0$. In Figure 1 we show the convergence of $\tau_{\varepsilon}$ for different discretizations. Moreover, we depict the evolution of $\left|\tau_{\varepsilon}-\tau^{*}\right|$ for the finest discretization, where we use as value for $\tau^{*}$ the optimal time for the smallest regularization parameter $\varepsilon_{i}$, i.e. $\tau^{*}=\tau_{\varepsilon_{i}}$. In Table 1 we report on the convergence of $\tau_{\varepsilon}$ and $\left\|\mathbf{y}_{\varepsilon}(1)-\mathbf{z}\right\|_{\mathbf{Y}^{0}}$ for the finest discretization. We observe the convergence rate

$$
\left|\tau_{\varepsilon}-\tau^{*}\right|=O(\varepsilon) .
$$


TABLE 1. Convergence history.

\begin{tabular}{cccc}
\hline$\varepsilon$ & $\tau_{\varepsilon}$ & $\left|\tau_{\varepsilon}-\tau^{*}\right|$ & $\left\|\mathbf{y}_{\varepsilon}(1)-\mathbf{z}\right\|_{\mathbf{Y}^{0}}$ \\
\hline $1.0000 \times 10^{-1}$ & $3.7339 \times 10^{-1}$ & $1.0692 \times 10^{-2}$ & $2.3722 \times 10^{-2}$ \\
$5.0000 \times 10^{-2}$ & $3.7802 \times 10^{-1}$ & $6.0621 \times 10^{-3}$ & $1.0028 \times 10^{-2}$ \\
$2.5000 \times 10^{-2}$ & $3.8024 \times 10^{-1}$ & $3.8405 \times 10^{-3}$ & $4.5856 \times 10^{-3}$ \\
$1.2500 \times 10^{-2}$ & $3.8233 \times 10^{-1}$ & $1.7519 \times 10^{-3}$ & $2.0920 \times 10^{-3}$ \\
$6.2500 \times 10^{-3}$ & $3.8344 \times 10^{-1}$ & $6.3668 \times 10^{-4}$ & $1.0169 \times 10^{-3}$ \\
$3.1250 \times 10^{-3}$ & $3.8408 \times 10^{-1}$ & & $5.1393 \times 10^{-4}$ \\
\hline
\end{tabular}
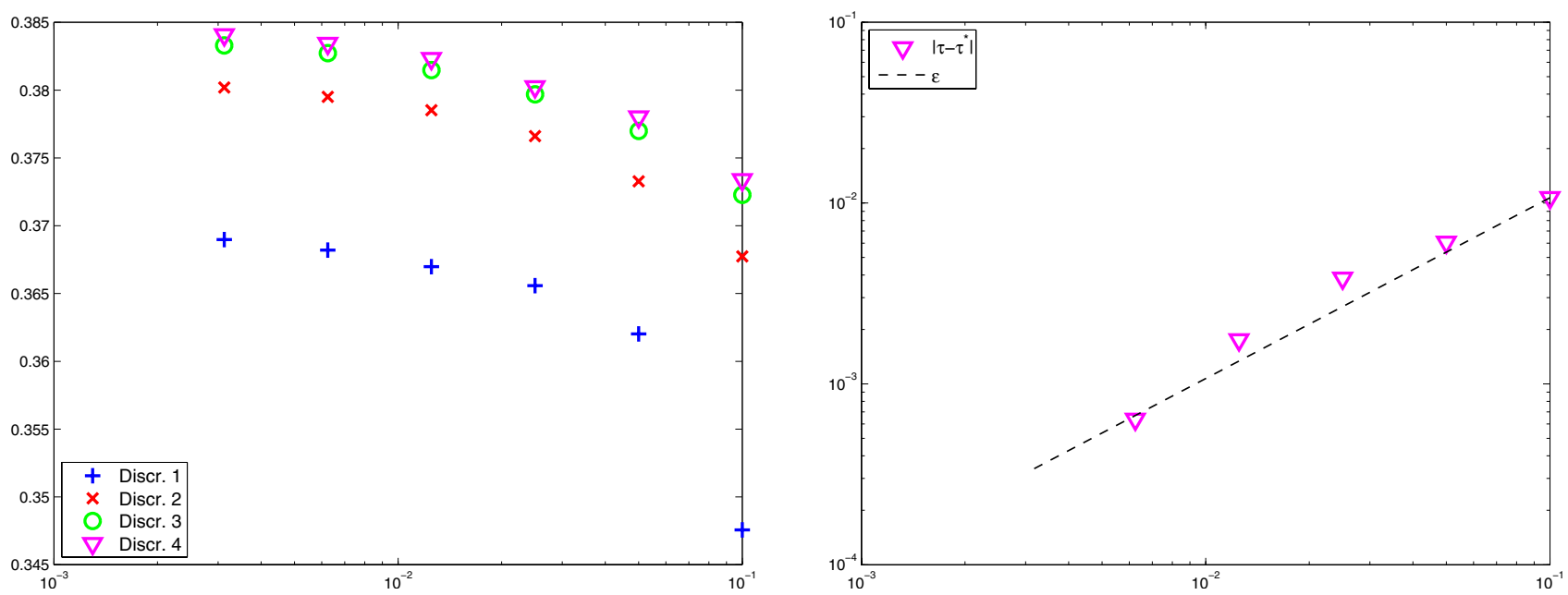

FiguRE 1. $\tau_{\varepsilon}$ vs. $\varepsilon$ for different discretizations; $\left|\tau_{\varepsilon}-\tau^{*}\right|$ vs. $\varepsilon$ for finest discretization.

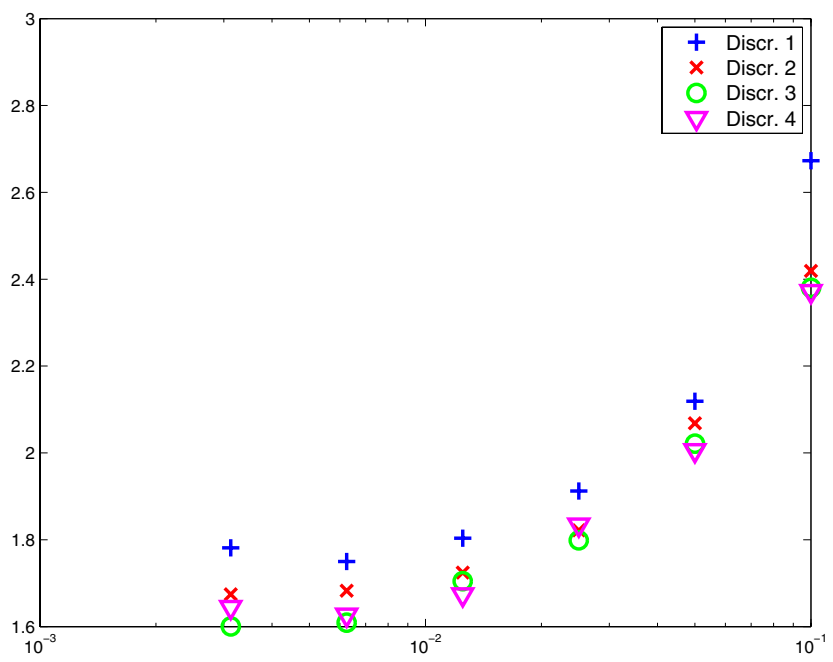

FiguRE 2. $\left\|\mathbf{p}_{\varepsilon}(1)\right\|_{\mathbf{P}^{1}}$ vs. $\varepsilon$ for different discretizations. 
As argued in Remark 4.5 this implies that

$$
\left\|\mathbf{y}_{\varepsilon}(1)-\mathbf{z}\right\|_{\mathbf{Y}^{0}}=O(\varepsilon)
$$

which can be seen in the table as well. This convergence rate implies that $\left\{\mathbf{p}_{\varepsilon}(1)\right\}$ is bounded in $\mathbf{P}^{1}$, where $\mathbf{p}_{\varepsilon}$ are the solutions of the undiscretized problem. In Figure 2, we plotted the evolution of $\left\|\mathbf{p}_{\varepsilon, h}(1)\right\|_{\mathbf{P}^{1}}$ for the solutions of the discrete problems for the 4 different discretizations, and we observe that the $\mathbf{P}^{1}$-norms of $\mathbf{p}_{\varepsilon, h}(1)$ are bounded uniformly with respect to $\varepsilon$ and with respect to the discretization. This suggests that the $a$-priori estimate of Theorem 4.4 is satisfied for this example, i.e. that (4.11) holds in the limit $\varepsilon \rightarrow 0$.

Acknowledgements. We are grateful to Prof. Hans-Josef Pesch for pointing out an error in an earlier version of the paper.

\section{REFERENCES}

[1] N.U. Ahmed, Finite-time null controllability for a class of linear evolution equations on a Banach space with control constraints. J. Optim. Theory Appl. 47 (1985) 129-158.

[2] V. Barbu. Optimal control of variational inequalities. Res. Notes Math. 100 (1984).

[3] D. Barcenas, H. Leiva and T. Maya, The transversality condition for infinite dimensional control systems. Revista Notas de Matematica 4 (2008) 25-36.

[4] C. Bardos, G. Lebeau and J. Rauch, Un exemple d'utilisation des notions de propagation pour le contrôle et la stabilisation de problèmes hyperboliques, Nonlinear hyperbolic equations in applied sciences. Rend. Sem. Mat. Univ. Politec. Torino 1988 (1989) 11-31.

[5] H.O. Fattorini, The time optimal problem for distributed control of systems described by the wave equation, in Control theory of systems governed by partial differential equations (Conf. Naval Surface Weapons Center, Silver Spring, Md., 1976). Academic Press, New York (1977) 151-175.

[6] H.O. Fattorini, Infinite-dimensional optimization and control theory. Cambridge University Press, Cambridge. Encyclopedia of Mathematics and its Applications. 62 (1999).

[7] H.O. Fattorini, Infinite dimensional linear control systems, The time optimal and norm optimal problems. Elsevier Science B.V., Amsterdam. North-Holland Mathematics Studies. 201 (2005).

[8] M. Gugat, Penalty techniques for state constrained optimal control problems with the wave equation. SIAM J. Control Optim. $48(2009 / 2010) 3026-3051$.

[9] M. Gugat and G. Leugering, $L^{\infty}$-norm minimal control of the wave equation: on the weakness of the bang-bang principle. ESAIM: COCV 14 (2008) 254-283.

[10] H. Hermes and J.P. LaSalle, Functional analysis and time optimal control. Academic Press, New York. Math. Sci. Eng. 56 (1969).

[11] K. Ito and K. Kunisch, Lagrange multiplier approach to variational problems and applications. Society for Industrial and Applied Mathematics (SIAM), Philadelphia, PA. Advances in Design and Control. 15 (2008).

[12] W. Krabs, On time-minimal distributed control of vibrating systems governed by an abstract wave equation. Appl. Math. Optim. 13 (1985) 137-149.

[13] W. Krabs, On time-minimal distributed control of vibrations. Appl. Math. Optim. 19 (1989) 65-73.

[14] I. Lasiecka, J.-L. Lions and R. Triggiani, Nonhomogeneous boundary value problems for second order hyperbolic operators. J. Math. Pures Appl. 65 (1986) 149-192.

[15] E.B. Lee and L. Markus, Foundations of optimal control theory. John Wiley \& Sons Inc., New York (1967).

[16] J.-L. Lions, Exact controllability, stabilization and perturbations for distributed systems. SIAM Rev. 30 (1988) 1-68.

[17] G. Peichl and W. Schappacher, Constrained controllability in Banach spaces. SIAM J. Control Optim. 24 (1986) $1261-1275$.

[18] K.D. Phung, G. Wang and X. Zhang, On the existence of time optimal controls for linear evolution equations. Discrete Contin. Dyn. Syst. Ser. B 8 (2007) 925-941.

[19] E. Zuazua, Propagation, observation, and control of waves approximated by finite difference methods. SIAM Rev. 47 (2005) 197-243. 